\title{
Wavespace-Based Coherent Deconvolution
}

\author{
Chris Bahr* \\ NASA Langley Research Center, Hampton, VA 23681 \\ christopher.j.bahr@nasa.gov \\ Louis Cattafesta ${ }^{\dagger}$ \\ Florida Center for Advanced Aero-Propulsion \\ Florida State University, Tallahassee, FL 32310
}

\begin{abstract}
Array deconvolution is commonly used in aeroacoustic analysis to remove the influence of a microphone array's point spread function from a conventional beamforming map. Unfortunately, the majority of deconvolution algorithms assume that the acoustic sources in a measurement are incoherent, which can be problematic for some aeroacoustic phenomena with coherent, spatially-distributed characteristics. While several algorithms have been proposed to handle coherent sources, some are computationally intractable for many problems while others require restrictive assumptions about the source field. Newer generalized inverse techniques hold promise, but are still under investigation for general use. An alternate coherent deconvolution method is proposed based on a wavespace transformation of the array data. Wavespace analysis offers advantages over curved-wave array processing, such as providing an explicit shift-invariance in the convolution of the array sampling function with the acoustic wave field. However, usage of the wavespace transformation assumes the acoustic wave field is accurately approximated as a superposition of plane wave fields, regardless of true wavefront curvature. The wavespace technique leverages Fourier transforms to quickly evaluate a shift-invariant convolution. The method is derived for and applied to ideal incoherent and coherent plane wave fields to demonstrate its ability to determine magnitude and relative phase of multiple coherent sources. Multiscale processing is explored as a means of accelerating solution convergence. A case with a spherical wave front is evaluated. Finally, a trailing edge noise experiment case is considered. Results show the method successfully deconvolves incoherent, partially-coherent, and coherent plane wave fields to a degree necessary for quantitative evaluation. Curved wave front cases warrant further investigation. A potential extension to nearfield beamforming is proposed.
\end{abstract}

\section{Nomenclature}

$\begin{array}{ll}\text { Dimensions } & \\ k_{x}, k_{y} & \text { Wavespace dimensions } \\ k_{\xi}, k_{\eta} & \text { Conjugate-power wavespace dimensions } \\ x, y & \text { Spatial dimensions } \\ \xi, \eta & \text { Conjugate-power spatial dimensions }\end{array}$

Symbols

a Relaxation parameter

$\boldsymbol{A} \quad$ Example system matrix

$\boldsymbol{B} \quad$ Matrix used in iteration stability analysis

C Matrix used in iteration stability analysis

*Research Aerospace Engineer, Aeroacoustics Branch, 2 North Dryden St., Mailstop 461, Member AIAA.

${ }^{\dagger}$ CSX Eminent Scholar and Professor, Department of Mechanical Engineering, FAMU-FSU College of Engineering, Associate Fellow AIAA. 


\begin{tabular}{|c|c|}
\hline$C F D$ & Acronym for Computational Fluid Dynamics \\
\hline$C S M$ & Acronym for Cross-Spectral Matrix \\
\hline$c_{0}$ & Isentropic speed of sound \\
\hline$D$ & Matrix used in iteration stability analysis \\
\hline$e$ & Vector of wavespace transform terms \\
\hline$E$ & Expected value operation \\
\hline$e r r$ & Normalized solution error \\
\hline$f$ & Temporal frequency \\
\hline FFT & Acronym for Fast Fourier Transform \\
\hline$G$ & Example cross-spectral matrix \\
\hline GPU & Acronym for Graphics Processor Unit \\
\hline $\boldsymbol{I}$ & Identity matrix \\
\hline$j$ & imaginary unit \\
\hline$k_{\max }$ & Maximum extent of wavespace grid \\
\hline M & Total number of microphones in array \\
\hline$n_{g}$ & Number of grid points in a beam map \\
\hline$n_{k}$ & Number of grid points in a given dimension in wavespace \\
\hline$n_{s}$ & Number of grid points in a given dimension required for linear convolution \\
\hline$p(x, y)$ & Sampled pressure field on array face \\
\hline$p_{m}$ & Pressure sampled at microphone $m$ for a given narrowband frequency \\
\hline$\tilde{p}\left(k_{x}, k_{y}\right)$ & Wavespace transform of sampled pressure field \\
\hline$\underset{\sim}{P}(\xi, \eta, x, y)$ & Spatial cross-spectrum \\
\hline$\tilde{P}\left(k_{\xi}, k_{\eta}, k_{x}, k_{y}\right)$ & Measured wavespace cross-spectrum \\
\hline$P S F$ & Array point spread function \\
\hline$q(x, y)$ & Continuous pressure field on array face \\
\hline$\underset{\sim}{\tilde{q}}\left(k_{x}, k_{y}\right)$ & Wavespace transform of true pressure field \\
\hline$\tilde{Q}\left(k_{\xi}, k_{\eta}, k_{x}, k_{y}\right)$ & Wavespace cross-spectrum of true pressure field \\
\hline$\hat{Q}$ & Forward transform of $\tilde{Q}$ \\
\hline$r_{\sigma}$ & Spectral radius of a matrix \\
\hline res & Solution residual \\
\hline$\tilde{R}\left(k_{\xi}, k_{\eta}, k_{x}, k_{y}\right)$ & Convolution of source field with array response \\
\hline$\hat{R}$ & Forward transform of $\tilde{R}$ \\
\hline$s(x, y)$ & Array sampling function in space \\
\hline$\tilde{s}\left(k_{x}, k_{y}\right)$ & Wavespace transform of array sampling function \\
\hline$\tilde{S}\left(k_{\xi}, k_{\eta}, k_{x}, k_{y}\right)$ & Wavespace cross-power sampling function \\
\hline$\hat{S}$ & Forward transform of $\tilde{S}$ \\
\hline$x$ & Vector of example sources \\
\hline$y$ & Vector of example observations \\
\hline$\Delta k$ & Grid point spacing in wavespace \\
\hline$\delta\left(\vec{x}-\vec{x}_{m}\right)$ & Dirac delta function \\
\hline$\Psi$ & Wavespace averaging function \\
\hline \multicolumn{2}{|c|}{ Subscripts and Superscripts } \\
\hline()$_{u}$ & Index of average bin in wavespace \\
\hline()$^{H}$ & Hermitian transpose \\
\hline()$^{(i)}$ & Current iteration number \\
\hline()$^{*}$ & Complex conjugate \\
\hline()$^{\prime}$ & Shifted coordinate \\
\hline$\overline{()}$ & Wavespace average \\
\hline
\end{tabular}




\section{Introduction}

$\mathrm{T}$ HE limitations of conventional beamforming impede the ability to extract quantitative information from traditional beam maps. Simple integration techniques can be used to provide approximate field values, but in general the inverse array problem of deconvolution must be solved to extract quantitative information from array data. Many frequency-domain deconvolution algorithms exist for incoherent source fields, for ex-

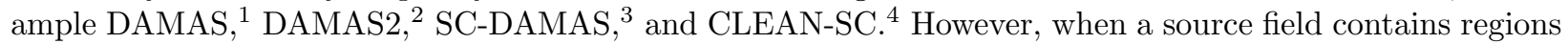
of coherence, there are fewer algorithm selections. Methods such as MACS are computationally reasonable, but assume a sparse source field ${ }^{5}$ Similarly, LORE is a quick algorithm but only solves for a subset of the scan grid ${ }^{[6}$ DAMAS-C has the potential to evaluate coherent sources over the entire scan grid of interest, but to do so is computationally challenging, even for small source fields ${ }^{7}$ Generalized inverse methods involving either eigenvalue subsets of the $C S M^{8}$ or the entire $C S M$ of an array data set ${ }^{9}$ may resolve distributed, partially-coherent source fields, but the range of applicability and limitations of these methods have yet to be completely addressed.

Deconvolution is fundamentally the inversion of a linear system, expressed in Eq. (1), with the application of constraints. For a vector of observations $\boldsymbol{y}$ and a matrix $\boldsymbol{A}$ modeling acoustic propagation, assuming the observations are uncontaminated by measurement noise, deconvolution attempts to solve

$$
\boldsymbol{y}=\boldsymbol{A x}
$$

for $\boldsymbol{x}$, representing the sources which pass through the system matrix to generate the observations. For many problems of interest, $\boldsymbol{A}$ is nearly singular, so direct inversion may not be possible, or yield a false solution. Algorithms such as DAMAS and DAMAS2 handle this inversion process iteratively, where an initial guess of $\boldsymbol{x}$ is multiplied by $\boldsymbol{A}$ and then subtracted from $\boldsymbol{y}$ to compute a residual. This residual is used to update the guess of $\boldsymbol{x}$. DAMAS2 makes a shift-invariant assumption, allowing the use of a Fourier-based technique to perform a fast convolution of $\boldsymbol{x}$ with $\boldsymbol{A}^{[2}$ Individual iterations of DAMAS2 are significantly faster than DAMAS for a given problem, but depending on the validity of the shift-invariant assumption and desired convergence criteria, more iterations of DAMAS2 may be required and accuracy may be limited. A smoothing filter may be used with DAMAS2 to favor certain solution sets and accelerate convergence.

Coherent deconvolution, where elements of $\boldsymbol{x}$ are statistically related to each other, is also modeled by Eq. (1). In this case, cross-spectral terms between grid points are included in $\boldsymbol{x}$ and $\boldsymbol{y}$, and $\boldsymbol{A}$ updated accordingly. Constraints on these additional elements are defined by a cross-spectral inequality 10 rather than a positivity constraint, and in general the terms will be complex. Unfortunately, scaling of general coherent deconvolution becomes problematic. If a beam map of interest has $n_{g}$ grid points, $\boldsymbol{x}$ and $\boldsymbol{y}$ each have $n_{g}^{2}$ elements, and $\boldsymbol{A}$ has $n_{g}^{4}$ elements. For example, a $20 \times 20$ beam map has $n_{g}=400$ grid points. This means $\boldsymbol{x}$ and $\boldsymbol{y}$ each have $20^{4}$ elements to account for all possible auto- and cross-spectral terms, meaning $\boldsymbol{A}$ has $20^{8}$, or 25.6 billion, elements. Conjugate-symmetry reduces the number of independent cross-spectral terms by a factor of two, but the scaling remains. If cross-spectral relationships between all of the grid points are considered, $\boldsymbol{A}$ is generally not sparse. While such a matrix of this size is possible to generate and store, it does not lend itself to efficient computation nor to more realistic grids.

To make the evaluation of $\boldsymbol{A x}$ more tractable for this form of deconvolution, it would be helpful to define the problem such that every element of $\boldsymbol{A}$ does not need to be computed and stored. Additionally, a fast convolution technique similar to that used by DAMAS2 is desired. A formulation of the coherent deconvolution process which does this is presented. A shift-invariance assumption, where the element of $\boldsymbol{A}$ relating a given element of $\boldsymbol{y}$ to an element of $\boldsymbol{x}$ is dependent only on the spatial separation of the two points, is applied to the coherence problem, just as with DAMAS2. This is done by analyzing the array data in wavenumber space, equivalent to plane wave beamforming. The analysis sacrifices the depth perception of nearfield beamforming due to the plane wave assumption.

The general problem of a transformation of a coherent pressure field to wavespace is first discussed in Section II. Basic implementation details are then addressed in Section III. Potential methods for accelerating convergence through faster computation or iteration count reduction are considered in Section IV. Section $\mathrm{V}$ presents results for different combinations of incoherent and coherent plane wave fields. Section VI evaluates a simulated curved wave field generated by a point source, as well as data from a trailing edge noise measurement. Finally, a method for nearfield beamforming involving the transformation of the deconvolved wavespace spectrum is proposed. 


\section{Formulation}

A formulation of the wavespace deconvolution problem which follows the form of Eq. (1) is necessary. Here, $\boldsymbol{y}$ is the set of array observations transformed to wavespace, and $\boldsymbol{A}$ is the model of the array sampling function. This array sampling function in wavespace is first constructed in regular space in terms of sampling theory, such that the true pressure field in space is multiplied by a distribution of delta functions corresponding to microphone locations in a planar array 11 This multiplication in the spatial domain becomes an equivalent convolution in the Fourier transform of the spatial domain, or the wavespace domain. The sampling function operating on the pressure field on a planar array face is given by

$$
s(x, y)=\sum_{m=1}^{M} \delta\left(x-x_{m}, y-y_{m}\right) .
$$

The sampled, temporally-Fourier transformed narrowband pressure field (frequency-dependence suppressed) is then given by

$$
\begin{aligned}
p(x, y) & =s(x, y) q(x, y) \\
& =\sum_{m=1}^{M} p_{m} \delta\left(x-x_{m}, y-y_{m}\right) .
\end{aligned}
$$

The 2-D spatial Fourier transforms of these quantities are thus

$$
\tilde{s}\left(k_{x}, k_{y}\right)=\iint s(x, y) e^{-j\left(k_{x} x+k_{y} y\right)} \mathrm{d} x \mathrm{~d} y=\frac{1}{M} \sum_{m=1}^{M} e^{-j\left(k_{x} x_{m}+k_{y} y_{m}\right)}
$$

and

$$
\begin{aligned}
\tilde{p}\left(k_{x}, k_{y}\right) & =\iint \tilde{s}\left(k_{x}-k_{x}^{\prime}, k_{y}-k_{y}^{\prime}\right) \tilde{q}\left(k_{x}^{\prime}, k_{y}^{\prime}\right) \mathrm{d} k_{x}^{\prime} \mathrm{d} k_{y}^{\prime} \\
& =\frac{1}{M} \sum_{m=1}^{M} p_{m} e^{-j\left(k_{x} x_{m}+k_{y} y_{m}\right)}
\end{aligned}
$$

where the normalization by $M$ is included such that a unit-magnitude plane wave in physical space has unit magnitude in wave space. Note that no $k_{z}$ transform is performed as all microphones are assumed co-planar.

Eq. (5) is a formulation of the basic wavespace sampling problem for a single block of data. To handle the stochastic nature of the pressure field, auto- and cross-powers are used to represent the ensemble-average characteristics of the field. A $C S M$ contains the auto- and cross-powers of the array microphones in the frequency domain ensemble-averaged across many blocks of data, and is represented by

$$
\boldsymbol{G}=\left[\begin{array}{cccc}
E\left[p^{*}\left(x_{1}, y_{1}\right) p\left(x_{1}, y_{1}\right)\right] & E\left[p^{*}\left(x_{1}, y_{1}\right) p\left(x_{2}, y_{2}\right)\right] & \ldots & E\left[p^{*}\left(x_{1}, y_{1}\right) p\left(x_{M}, y_{M}\right)\right] \\
E\left[p^{*}\left(x_{2}, y_{2}\right) p\left(x_{1}, y_{1}\right)\right] & E\left[p^{*}\left(x_{2}, y_{2}\right) p\left(x_{2}, y_{2}\right)\right] & \ldots & E\left[p^{*}\left(x_{2}, y_{2}\right) p\left(x_{M}, y_{M}\right)\right] \\
\vdots & \vdots & \ddots & \vdots \\
E\left[p^{*}\left(x_{M}, y_{M}\right) p\left(x_{1}, y_{1}\right)\right] & E\left[p^{*}\left(x_{M}, y_{M}\right) p\left(x_{2}, y_{2}\right)\right] & \ldots & E\left[p^{*}\left(x_{M}, y_{M}\right) p\left(x_{M}, y_{M}\right)\right]
\end{array}\right] .
$$

Note that the 2-D CSM contains 4-D spatial covariance information,

$$
P(\xi, \eta, x, y)=E\left[p^{*}(\xi, \eta) p(x, y)\right] .
$$

The $C S M$ could be directly transformed to wavespace through a 4-D Fourier transform,

$$
\mathfrak{F}\{P(\xi, \eta, x, y)\}=\iiint \int P(\xi, \eta, x, y) e^{-j\left(k_{\xi} \xi+k_{\eta} \eta+k_{x} x+k_{y} y\right)} \mathrm{d} \xi \mathrm{d} \eta \mathrm{d} x \mathrm{~d} y .
$$

However, for this discussion the wavespace cross-spectrum is the desired quantity, which is defined by

$$
\tilde{P}\left(k_{\xi}, k_{\eta}, k_{x}, k_{y}\right)=E\left[\tilde{p}^{*}\left(k_{\xi}, k_{\eta}\right) \tilde{p}\left(k_{x}, k_{y}\right)\right] .
$$


This can be determined by substituting the first line of Eq. (5) into Eq. (9),

$$
\begin{aligned}
\tilde{P}\left(k_{\xi}, k_{\eta}, k_{x}, k_{y}\right)= & E\left[\tilde{p}^{*}\left(k_{\xi}, k_{\eta}\right) \tilde{p}\left(k_{x}, k_{y}\right)\right] \\
= & E\left[\iint \tilde{s}^{*}\left(k_{\xi}-k_{\xi}^{\prime}, k_{\eta}-k_{\eta}^{\prime}\right) \tilde{q}^{*}\left(k_{\xi}^{\prime}, k_{\eta}^{\prime}\right) \mathrm{d} k_{\xi}^{\prime} \mathrm{d} k_{\eta}^{\prime} \times\right. \\
& \left.\iint \tilde{s}\left(k_{x}-k_{x}^{\prime}, k_{y}-k_{y}^{\prime}\right) \tilde{q}\left(k_{x}^{\prime}, k_{y}^{\prime}\right) \mathrm{d} k_{x}^{\prime} \mathrm{d} k_{y}^{\prime}\right] \\
= & \iiint \int \tilde{s}^{*}\left(k_{\xi}-k_{\xi}^{\prime}, k_{\eta}-k_{\eta}^{\prime}\right) \tilde{s}\left(k_{x}-k_{x}^{\prime}, k_{y}-k_{y}^{\prime}\right) \times \\
& E\left[\tilde{q}^{*}\left(k_{\xi}^{\prime}, k_{\eta}^{\prime}\right) \tilde{q}\left(k_{x}^{\prime}, k_{y}^{\prime}\right)\right] \mathrm{d} k_{\xi}^{\prime} \mathrm{d} k_{\eta}^{\prime} \mathrm{d} k_{x}^{\prime} \mathrm{d} k_{y}^{\prime} \\
= & \iiint \int \tilde{S}\left(k_{\xi}-k_{\xi}^{\prime}, k_{\eta}-k_{\eta}^{\prime}, k_{x}-k_{x}^{\prime}, k_{y}-k_{y}^{\prime}\right) \tilde{Q}\left(k_{\xi}^{\prime}, k_{\eta}^{\prime}, k_{x}^{\prime}, k_{y}^{\prime}\right) \mathrm{d} k_{\xi}^{\prime} \mathrm{d} k_{\eta}^{\prime} \mathrm{d} k_{x}^{\prime} \mathrm{d} k_{y}^{\prime} .
\end{aligned}
$$

This shows that the measured wavespace cross-spectrum is the convolution of the wavespace cross-power sampling function $\tilde{S}$ and the true wavespace cross-spectrum, $\tilde{Q}$.

The measured wavespace cross-spectrum $\tilde{P}$ can be computed by substituting the second line of Eq. (5) into Eq. (9), giving

$$
\begin{aligned}
\tilde{P}\left(k_{\xi}, k_{\eta}, k_{x}, k_{y}\right) & =E\left[\tilde{p}^{*}\left(k_{\xi}, k_{\eta}\right) \tilde{p}\left(k_{x}, k_{y}\right)\right] \\
& =E\left[\frac{1}{M} \sum_{n=1}^{M} p_{n}^{*} e^{j\left(k_{\xi} \xi_{n}+k_{\eta} \eta_{n}\right)} \frac{1}{M} \sum_{m=1}^{M} p_{m} e^{-j\left(k_{x} x_{m}+k_{y} y_{m}\right)}\right] \\
& =\frac{1}{M^{2}} \sum_{n=1}^{M} \sum_{m=1}^{M} E\left[p_{n}^{*} p_{m}\right] e^{j\left(k_{\xi} \xi_{n}+k_{\eta} \eta_{n}\right)} e^{-j\left(k_{x} x_{m}+k_{y} y_{m}\right)} .
\end{aligned}
$$

This is similar to the derivation given by Capor ${ }^{12}$ which relates the cross-spectrum between two points to their separation in physical space and the wavespace spectrum, but in a form of a forward transform instead of an inverse. As a $4 \mathrm{D}$ representation of $2 \mathrm{D}$ wavespace, this representation captures the coherence relationship between wavespace locations. The term $E\left[p_{n}^{*} p_{m}\right]$ in Eq. 111 is an entry in the CSM. Thus, the double-summation can be re-expressed as

$$
\tilde{P}\left(k_{\xi}, k_{\eta}, k_{x}, k_{y}\right)=\frac{1}{M^{2}} \boldsymbol{e}^{H} \boldsymbol{G e},
$$

with the Hermitian transpose of $\boldsymbol{e}$ given by

$$
\boldsymbol{e}^{H}=\left[\begin{array}{llll}
e^{j\left(k_{\xi} \xi_{1}+k_{\eta} \eta_{1}\right)} & e^{j\left(k_{\xi} \xi_{2}+k_{\eta} \eta_{2}\right)} & \ldots & e^{j\left(k_{\xi} \xi_{M}+k_{\eta} \eta_{M}\right)}
\end{array}\right] .
$$

Similarly, the wavespace cross-power sampling function can be constructed as

$$
\tilde{S}\left(k_{\xi}, k_{\eta}, k_{x}, k_{y}\right)=\frac{1}{M^{2}} e^{H}\left[\begin{array}{cccc}
1 & 1 & \ldots & 1 \\
1 & 1 & \ldots & 1 \\
\vdots & \vdots & \ddots & \vdots \\
1 & 1 & \ldots & 1
\end{array}\right] \boldsymbol{e}
$$

Note that when diagonal removal13 is applied, the normalization should change from $M^{2}$ to $M^{2}-M$ to maintain unit response in the transformation as $m=n$ terms are neglected.

As previously stated, formulation of the problem is desired which follows Eq. (1). In this section, a model is constructed in which $\tilde{P}$ contains the information in the observation vector $\boldsymbol{y}$, and $\tilde{S}$ contains the information in the system matrix $\boldsymbol{A}$. Taking the source field $\tilde{Q}$ as the solution vector $\boldsymbol{x}$, the problem of interest is to find the source field which best fits the data in the observation vector, obeying the given constraints and avoiding explicit computation or storage of the entirety of $\boldsymbol{A}$.

\section{Implementation}

To solve this problem, $\tilde{P}$ and $\tilde{S}$ are first constructed for a discrete set of coordinates in wavespace. For simplicity, a hypercube grid is considered, with each coordinate set spanning $k_{\xi}=k_{\eta}=k_{x}=k_{y}=$ 
[- $\left.k_{\max }: \Delta k: k_{\max }\right]$ for a total grid size of $n_{k} \times n_{k} \times n_{k} \times n_{k}$. The selection of the parameters $k_{\max }, \Delta k$ and $n_{k}$ are discussed subsequently, but are dependent on the array, the problem of interest and available computational resources.

An iterative solution of Eq. (1) can take the form of

$$
\boldsymbol{x}^{(i+1)}=\boldsymbol{x}^{(i)}+\frac{1}{a}\left(\boldsymbol{y}-\boldsymbol{A} \boldsymbol{x}^{(i)}\right) .
$$

Constraints are enforced after every iteration. The general constraints considered for DAMAS-C are adapted ${ }^{7}$ A real positivity constraint is applied to autospectral terms,

$$
\begin{aligned}
& \Re\left\{\tilde{Q}\left(k_{x}, k_{y}, k_{x}, k_{y}\right)\right\} \geq 0 \\
& \Im\left\{\tilde{Q}\left(k_{x}, k_{y}, k_{x}, k_{y}\right)\right\}=0 .
\end{aligned}
$$

Cross-spectral terms must obey the cross-spectral inequality and follow appropriate conjugate behavior,

$$
\begin{aligned}
\left|\tilde{Q}\left(k_{\xi}, k_{\eta}, k_{x}, k_{y}\right)\right|^{2} & \leq \tilde{Q}\left(k_{x}, k_{y}, k_{x}, k_{y}\right) \tilde{Q}\left(k_{\xi}, k_{\eta}, k_{\xi}, k_{\eta}\right) \\
\tilde{Q}\left(k_{x}, k_{y}, k_{\xi}, k_{\eta}\right) & =\tilde{Q}^{*}\left(k_{\xi}, k_{\eta}, k_{x}, k_{y}\right) .
\end{aligned}
$$

After each iteration, autospectral constraints are applied first, followed by cross-spectral constraints.

The algorithm takes the same structure as that used with DAMAS2, where the equivalent evaluation of $\boldsymbol{A} \boldsymbol{x}^{(i)}$ from Eq. (15) is computed as a function evaluation through the use of Fourier transforms, rather than an explicit linear algebra operation.$^{2}$ Unlike DAMAS2, no regularization filter is applied at this stage of research, as the effect this may have on phase relationships is unclear.

1. Forward-transform $\tilde{S}$ to $\hat{S}$ via a 4 -D transform like Eq. (8).

2. Initialize $\tilde{Q}$ to zeroes.

3. Iterate (to convergence or maximum desired function evaluations)

- Forward transform $\tilde{Q}^{(i)}$ to $\hat{Q}$, again using Eq. (8).

- Compute the product $\hat{R}=\hat{S} \hat{Q}$.

- Inverse transform $\hat{R}$ to $\tilde{R}$ to obtain the effective convolution of $\tilde{S}$ with $\tilde{Q}^{(i)}$.

- Update $\tilde{Q}^{(i+1)}=\tilde{Q}^{(i)}+\frac{1}{a}(\tilde{P}-\tilde{R})$.

- Enforce constraints.

Note that for linear convolution, $\tilde{Q}$ must be zero-padded, and $\tilde{S}$ must be constructed on the larger, padded grid scale. Here, the grid coordinates all span [-2k $\left.k_{\max }: \Delta k: 2 k_{\max }\right]$, for a total grid size of $n_{s} \times n_{s} \times n_{s} \times n_{s}$. If $n_{k}$ is selected to be odd such that the plane wave bin $\tilde{Q}(0,0,0,0)$ is in the solution set, then $n_{s}=2 n_{k}-1$ and the total grid size is $\left(2 n_{k}-1\right)^{4}$, or approximately 16 times the size of the baseline grid. $\tilde{Q}$ must be padded to this size prior to convolution, and the appropriate subset of grid points selected in the $\tilde{P}-\tilde{R}$ step of the algorithm.

The relaxation parameter, $a$, is a critical component of the algorithm, as the iterative update is completely unstable without it. With DAMAS2, this parameter is specified as the sum of the absolute value of the 2D array response within the baseline grid domain. Extended to this $4 \mathrm{D}$ problem, it is computed by

$$
a=\sum_{k_{\xi}=-k_{\max }}^{k_{\max }} \sum_{k_{\eta}=-k_{\max }}^{k_{\max }} \sum_{k_{x}=-k_{\max }}^{k_{\max }} \sum_{k_{y}=-k_{\max }}^{k_{\max }}\left|\tilde{S}\left(k_{\xi}, k_{\eta}, k_{x}, k_{y}\right)\right| .
$$

This value is effectively the maximum row sum of absolute values of the $\boldsymbol{A}$ representation of $\tilde{S}$ in Eq. (15). This corresponds to the matrix norm $\|\boldsymbol{A}\|_{1} \cdot{ }^{14}$ As the spectral radius of a square matrix must be less than or equal to its operator norms, normalizing the iterative process by this norm is a conservative way to stabilize the solution procedure. 
While this algorithm bypasses explicit storage of $\boldsymbol{A}$, which would have a size of $n_{k}^{8}$, problem scaling can still be an issue. Specifically, $n_{s}^{4}$ problem scaling is still non-trivial. For example, if $n_{k}=25$, each $n_{s}^{4}$-sized array, where $n_{s}=49$, contains 5.8 million elements. While this has moderate storage requirements, $46 \mathrm{MB}$ for single-precision complex, it is still a significant amount of data to handle with an algorithm which may require a significant number of iterations. Hardware-tuned FFT algorithms ${ }^{15}$ can help mitigate this expense, but the cost of a given problem size is still a driving concern, since based on Eq. (18), larger problems will in general require greater relaxation parameters for a given array resolution.

Previous research ${ }^{1}$ indicates that in deconvolution the grid spacing should be between approximately $5 \%$ and $20 \%$ of the sampling pattern's 3 -dB main lobe. In wavespace, this sets an effective maximum value for $\Delta k$ for a given array. The maximum wavenumber $k_{\max }$ of interest is highly problem-dependent. In a limiting case of plane waves arriving within a limited cone of directions, $k_{\max }$ can simply be selected to properly encompass the appropriate region within wavespace. However, for a general problem where no information is known about the acoustic field $k_{\max }$ will need to meet, if not exceed, the acoustic wavenumber, $2 \pi f / c_{0}$ $(\mathrm{rad} / \mathrm{m})$, or $f / c_{0}\left(\mathrm{~m}^{-1}\right)$. These constraints, combined with the aforementioned scaling issue, currently limit the method to either problems with limited direction of arrival, or low frequencies. For example, the $0.74 \mathrm{~m}$ aperture outer array used at the University of Florida Aeroacoustic Flow Facility (UFAFF) ${ }^{16}$ can adequately capture the entire acoustic radiation circle with a $\Delta k$ of $17 \%$ of the sampling pattern's 3 -dB main lobe, 2 $\mathrm{m}^{-1}$, at $f=1 \mathrm{kHz}$ with $n_{k}=17$. An individual transform of a grid required for this scale is reasonably quick, and thus can be considered for analysis. Higher frequencies become an issue, as acoustic wavenumber scales linearly with frequency. To maintain a given $\Delta k$, this means that $n_{k}$ must also scale linearly with frequency, so the overall problem scales as the fourth power of frequency. Similarly, extending $k_{\text {max }}$ beyond the acoustic wavenumber to evaluate subsonic hydrodynamic contamination can only be considered for very low frequencies.

As formulated above and tested, the algorithm worked for simple simulated problems. However, for a grid with $n_{k}=9$, approximately 500,000 iterations were required to converge for an ideal point source, defined by

$$
e r r^{(i+1)}=\|\tilde{P}-\tilde{R}\| .
$$

The residual change in solution is also followed, defined by,

$$
r e s^{(i+1)}=\left\|\tilde{Q}^{(i+1)}-\tilde{Q}^{(i)}\right\|,
$$

Numeric convergence is achieved when the error reaches the minimum unit-round based on floating-point single precision. ${ }^{14}$ For larger grids, numeric convergence never occurred, although reasonable-looking solutions were achieved. For these, the residual was tracked until it became small relative to the solution magnitude. Changing to double-precision did not appear to improve convergence behavior for the $n_{k}=9$ grids, and came with a significant run time and memory cost. At this stage of research, the effect of floating-point precision on the convergence behavior of larger grids was not considered.

\section{Solution Acceleration}

To improve the utility of this deconvolution technique, acceleration methods must be explored. Three possibilities are considered: reducing the operating time of an individual iteration, using a more aggressive relaxation parameter $a$, and improving the initial solution guess $\tilde{Q}^{(0)}$.

\section{Computation Time}

A direct reduction in iteration time is easiest to consider, as 500,000 iterations may appear reasonable if the total code run time is 10 seconds. Hardware-optimized FFT algorithms are continuously improving, and some companies are now offering pre-made libraries for GPU computing, ${ }^{17}$ potentially providing significant performance improvements. Additionally, pruned FFT methods can be considered. The majority of the elements in $\tilde{Q}$ are zeros to meet padding requirements. The majority of the elements in $\tilde{R}$ are thrown away prior to subtraction from $\tilde{P}$. Hardware implementations of pruned FFTs exist $\frac{18}{18}$ and may provide significant benefits in cases of 4-dimensional zero-padding. However, neither these nor GPU methods are explored at this stage of the research.

One potential algorithm was considered. The 4-dimensional FFT is, as previously mentioned, a fast mechanism for evaluating the $\boldsymbol{A} \boldsymbol{x}^{(i)}$ term from Eq. 15 without storing the entirety of $\boldsymbol{A}$. Alternative fast 
convolution techniques exist. One such technique takes advantage of the fact that, with appropriate element ordering, $\boldsymbol{A}$ has a multilevel block-Toeplitz structure. It is possible to store only the unique elements of this matrix in a vector and perform a fast convolution with an appropriately-padded $\boldsymbol{x}$, providing an alternative storage-free fast convolution. $\frac{19}{19}$ The algorithm as cited was implemented, with some modifications to the zero-padding code and element access for performance. It was found to perform slightly better for some grid sizes and slightly worse for others. While it may hold some promise for improvement, the method is not further explored here.

\section{Relaxation}

The relaxation parameter computed in Eq. (18) is a conservative one. With some effort, a more aggressive parameter which maintains stability may be derived, following the method presented by Atkinson! ${ }^{14}$ Eq. (1) can be re-structured by splitting $\boldsymbol{A}$,

$$
\boldsymbol{A}=\boldsymbol{B}-\boldsymbol{C},
$$

and re-writing as

$$
B \boldsymbol{x}=\boldsymbol{y}+\boldsymbol{C x} .
$$

The iterative method is thus given as

$$
\boldsymbol{B} \boldsymbol{x}^{(i+1)}=\boldsymbol{y}+\boldsymbol{C} \boldsymbol{x}^{(i)}
$$

Matrix $\boldsymbol{D}$ can be defined as

$$
\boldsymbol{D}=\boldsymbol{B}^{-1} \boldsymbol{C} .
$$

The stability criterion for the (unconstrained) iteration method is then given as

$$
r_{\sigma}(\boldsymbol{D})<1 .
$$

With some rearrangement, Eq. 15 yields $\boldsymbol{B}=a \boldsymbol{I}$ and $\boldsymbol{C}=a \boldsymbol{I}-\boldsymbol{A}$. This gives $\boldsymbol{D}=\boldsymbol{I}-\frac{1}{a} \boldsymbol{A}$. Since $r_{\sigma}(\boldsymbol{D})$ is the magnitude of the largest eigenvalue of $\boldsymbol{D}$, finding this eigenvalue and iterating $a$ until the spectral radius drops below unity should yield a more aggressive value for $a$. This may appear problematic at first as the goal of this method is to avoid computing and storing matrices of size $\boldsymbol{A}$, which would be necessary to determine all of the eigenvalues of $\boldsymbol{D}$. However, there are methods for computing the largest eigenvalue of $\boldsymbol{D}$ as a function call evaluating the matrix vector product $\boldsymbol{D} \boldsymbol{x}$ for a given input vector $\boldsymbol{x}$, rather than using $\boldsymbol{D}$ itself. Specifically, the MATLAB function eigs can output the largest eigenvalue of a large, dense, non-symmetric complex matrix modeled by a function call. As used with these function calls, eigs fails to converge when the eigenvalue of $\boldsymbol{D}$ drops below unity, so the smallest value of $a$ for which eigs fails to give a converged output is used. For this study, the value varies between one-half and one-twentieth of the value given by Eq. 18 for the modeled arrays and grids, and appears stable for the cases considered. The reduced relaxation parameter significantly improves the convergence behavior of the algorithm. Cases with $n_{k}=9$ reach acceptable convergence in 50,000 iterations rather than 500,000.

\section{Initial Guess}

Additional improvement is desired for larger grids. An improved initial guess can have a significant impact on the direction and rate of convergence of the method, and is worth investigating. A variety of initialization methods were considered (e.g. Wiener filtering, initializing with DAMAS3, 2 starting from $\tilde{P}$ ). However, these techniques yielded behavior generally worse than starting with $\tilde{Q}=0$. However, one method which did show improvement for some situations is multiscale analysis. Analyzing problems on multiple scales is common in many fields such as $\mathrm{CFD}^{20}$ and image processing 21 ?22 Full multigrid solution methods have even been applied to seismic data deconvolution. ${ }^{23}$ Currently, only the simple case is explored, whereby coarse scales are used to initialize fine scales.

The construction of scales for deconvolution analysis requires some care. If the coarse grids have very few points per beamwidth, the deconvolution results may be unreliable. This can be due to both the limited resolution of the array response's main lobe, as well as the discrete sampling pattern missing side lobes. In an attempt to mitigate this coarse-grid limitation, formulations for an average wavespace pressure field and average array response are derived and used instead of those given by Eqs. (12) and (14). 
For the moment, consider a one-dimensional wavespace transform of the pressure field measured by a linear array,

$$
\tilde{p}\left(k_{x}\right)=\frac{1}{M} \sum_{m=1}^{M} p_{m} e^{-j k_{x} x_{m}} .
$$

For a grid spacing $\Delta k$, the average value of the wavespace transform at grid point $k_{x, u}$ is

$$
\begin{aligned}
\overline{\tilde{p}}\left(k_{x, u}\right) & =\frac{1}{\Delta k} \int_{k_{x, u}-\Delta k / 2}^{k_{x, u}+\Delta_{k} / 2} \frac{1}{M} \sum_{m=1}^{M} p_{m} e^{-j k_{x} x_{m}} \mathrm{~d} k_{x} \\
& =\frac{1}{M} \sum_{m=1}^{M} p_{m} \int_{k_{x, u}-\Delta k / 2}^{k_{x, u}+\Delta_{k} / 2} \frac{e^{-j k_{x} x_{m}}}{\Delta k} \mathrm{~d} k_{x} . \\
& =\frac{1}{M} \sum_{m=1}^{M} p_{m} \Psi\left(k_{x, u}, \Delta k, x_{m}\right)
\end{aligned}
$$

When $x_{m}=0, \Psi=1$. When $x_{m} \neq 0$,

$$
\begin{aligned}
\Psi\left(k_{x, u}, \Delta k, x_{m}\right) & =\left.\frac{1}{-j x_{m} \Delta k} e^{-j k_{x} x_{m}}\right|_{k_{x, u}-\Delta k / 2} ^{k_{x, u}+\Delta k / 2} \\
& =\frac{1}{-j x_{m} \Delta k}\left[e^{-j x_{m}\left(k_{x, u}+\Delta k / 2\right)}-e^{-j x_{m}\left(k_{x, u}-\Delta k / 2\right)}\right] .
\end{aligned}
$$

Using the definition from Eq. 28, the average 4-dimensional wavespace cross-spectrum is constructed as

$$
\begin{aligned}
\overline{\tilde{P}}\left(k_{\xi, u}, k_{\eta, u}, k_{x, u}, k_{y, u}\right)= & \frac{1}{M^{2}} \sum_{n=1}^{M} \sum_{m=1}^{M} E\left[p_{n}^{*} p_{m}\right] \times \\
& \Psi\left(k_{\xi, u}, \Delta k,-\xi_{n}\right) \Psi\left(k_{\eta, u}, \Delta k,-\eta_{n}\right) \Psi\left(k_{x, u}, \Delta k, x_{m}\right) \Psi\left(k_{y, u}, \Delta k, y_{m}\right),
\end{aligned}
$$

where the negative sign on the conjugate-dimension coordinates in $\Psi$ accounts for the conjugate phase of the integration. Similarly, the average cross-power sampling function is given by

$$
\begin{aligned}
\overline{\tilde{S}}\left(k_{\xi, u}, k_{\eta, u}, k_{x, u}, k_{y, u}\right)= & \frac{1}{M^{2}} \sum_{n=1}^{M} \sum_{m=1}^{M} \\
& \Psi\left(k_{\xi, u}, \Delta k,-\xi_{n}\right) \Psi\left(k_{\eta, u}, \Delta k,-\eta_{n}\right) \Psi\left(k_{x, u}, \Delta k, x_{m}\right) \Psi\left(k_{y, u}, \Delta k, y_{m}\right) .
\end{aligned}
$$

As with the non-averaged formulations, the $M^{2}$ denominator term is replaced with $M^{2}-M$ when diagonal removal is applied and $m=n$ terms neglected. As a final correction, both $\overline{\tilde{P}}$ and $\overline{\tilde{S}}$ are normalized by $\overline{\tilde{S}}(0,0,0,0)$, since this value is no longer unity except for very fine grids (where Eqs. 29$)$ and $(30)$ collapse to Eqs. (12) and (14), respectively). As the correction is effectively applied to both sides of Eq. (1), equality of the equivalent system of equations is maintained.

With modified equations defined, a means of transitioning from more coarse grids to more fine grids must now be determined. If some knowledge of the source field $\tilde{Q}$ is available a-priori, a model-based interpolation scheme would be preferable. However, in constructing a general deconvolution method, such knowledge cannot be assumed. For example, in the case of discrete plane waves, the source field is discontinuous. To mitigate the influence of discontinuities on blind interpolation, a linear interpolation scheme is considered. To account for potential incoherent source regions in the interpolation scheme, autospectral terms of $\tilde{Q}$ are upscaled separately from cross-spectral terms. This interpolation dependency is shown schematically for a two-dimensional conjugate-power case in Figure 1, where a $3 \times 3$ grid is upscaled to a $5 \times 5$ grid. In this representation, the wavespace autospectrum exists on the grid diagonal, with cross-spectral terms elsewhere. Note that conjugate symmetry exists between the upper and lower triangular portions of the grid.

\section{Plane Wave Results}

Results for simulated plane wave fields, which are identically shift-invariant, are presented. Fields of varying complexity are considered. All simulations use the microphone layout of the outer UFAFF array $\underline{\underline{16}}$ 


\begin{tabular}{|c|c|c|c|c|c|}
\hline & $k_{x}=1$ & $k_{x}=2$ & $k_{x}=3$ & $k_{x}=4$ & $k_{x}=5$ \\
\hline$k_{\xi}=1$ & $Q(1,1)$ & $\cdot$ & $Q(1,3)$ & $\cdot$ & $Q(1,5)$ \\
\hline$k_{\xi}=2$ & $\cdot$ & $\cdot$ & $\cdot$ & $\cdot$ & $\cdot$ \\
\hline$k_{\xi}=3$ & $Q(3,1)$ & $\cdot$ & $Q(3,3)$ & $\cdot$ & $Q(3,5)$ \\
\hline$k_{\xi}=4$ & $\cdot$ & $\cdot$ & $\cdot$ & $\cdot$ & $\cdot$ \\
\hline$k_{\xi}=5$ & $Q(5,1)$ & $\cdot$ & $Q(5,3)$ & $\cdot$ & $Q(5,5)$ \\
\hline
\end{tabular}

(a) Pre-interpolation grid

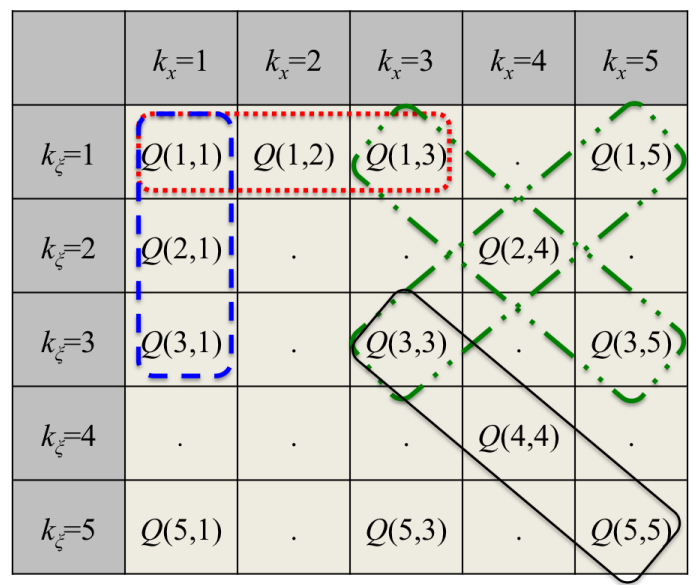

(b) Interpolation dependencies

Figure 1. Linear grid interpolation for 2-D problem upscaling.

mentioned in Section III. Details of the array can be found in the reference, but in brief the array is a 0.74 $\mathrm{m}$ aperture, 5-arm log spiral based on the methodology of Underbrink ${ }^{24}$ consisting of 40 total microphones. The array's average autospectrum sampling function $\tilde{S}\left(k_{x}, k_{y}, k_{x}, k_{y}\right)$ is shown for two different resolutions in Figure 2 The 3-dB beamwidth of the autospectrum of the sampling function is $1.47 \mathrm{~m}^{-1}$. The coarse grid with $n_{s}=17$ points thus has 3 points per beamwidth, while the fine grid with $n_{s}=33$ points has 5 points per beamwidth. Diagonal removal is applied for all cases. For array response plots, the color scale is in $\mathrm{dB}$ referenced to the array response center value of unity. For plots of pressure and source spectra, the color scale is in $\mathrm{dB}$ ref $20 \mu \mathrm{Pa}$. As pressure autospectra can contain negative values with diagonal removal, these values are set to $-\infty \mathrm{dB}$ for plotting.

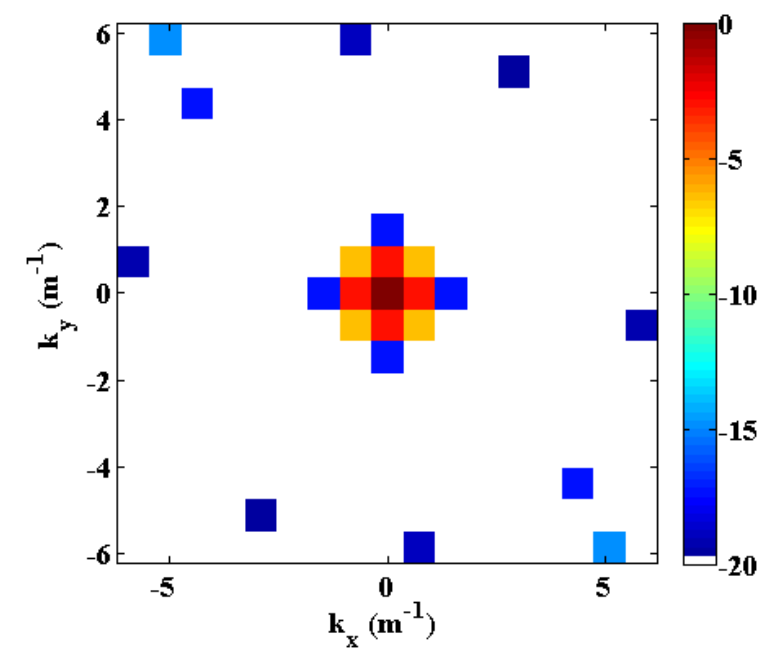

(a) $n_{s}=17$ points

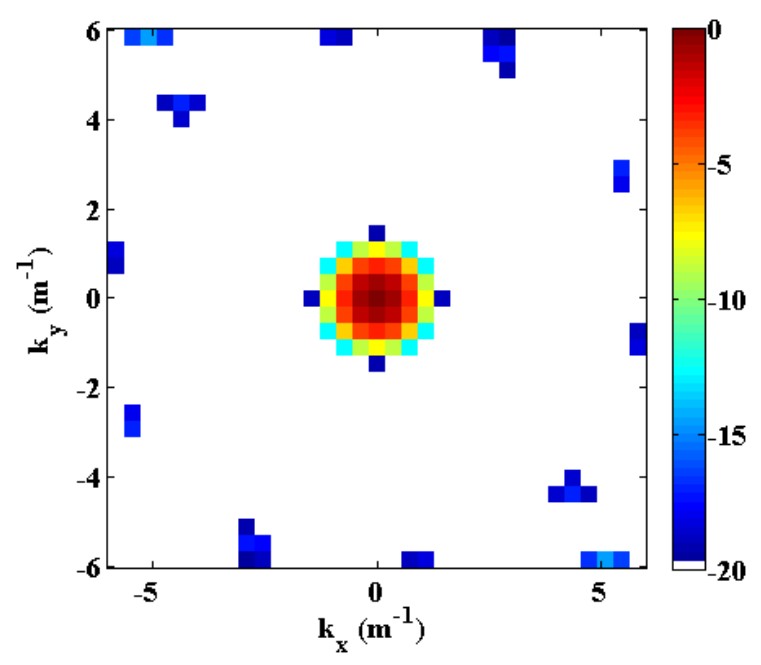

(b) $n_{s}=33$ points

Figure 2. Autospectrum of average sampling function $\overline{\widetilde{S}}$ for two different resolutions.

\section{Isolated Plane Wave}

The first case considered is a single, broadside plane wave, corresponding to a point source in wavespace with $k_{x}=0 \mathrm{~m}^{-1}$ and $k_{y}=0 \mathrm{~m}^{-1}$. The plane wave is simulated with an amplitude of $100 \mathrm{~dB}$. The simulation parameters use the more coarse sampling grid from Figure 2a, corresponding to $n_{k}=9$ points and $n_{s}=17$ 
points, with $k_{\max }=2.92 \mathrm{~m}^{-1}$. The average wavespace auto-spectrum $\overline{\tilde{P}}\left(k_{x}, k_{y}, k_{x}, k_{y}\right)$ for this case is plotted in Figure $3 \mathrm{a}$. As expected for a centered point source, it appears as a scaled version of the average sampling function. The average cross-spectrum magnitude $\left|\tilde{P}\left(0,0, k_{x}, k_{y}\right)\right|$ is shown in Figure $3 \mathrm{~b}$.

After 10,000 iterations, single-precision convergence as defined by Eq. (19) occurs, requiring 100 seconds of runtime on a $2.2 \mathrm{GHz}$ Core i7 MacBook Pro for code utilizing the FFTW engine present in MATLAB 2011b. The resultant auto- and cross-spectra, $\tilde{Q}\left(k_{x}, k_{y}, k_{x}, k_{y}\right)$ and $\left|\tilde{Q}\left(0,0, k_{x}, k_{y}\right)\right|$, are shown in Figures $3 \mathrm{c}$ and $3 \mathrm{~d}$. As expected, only one source is observed in the spectrum and no cross-powers are evident.

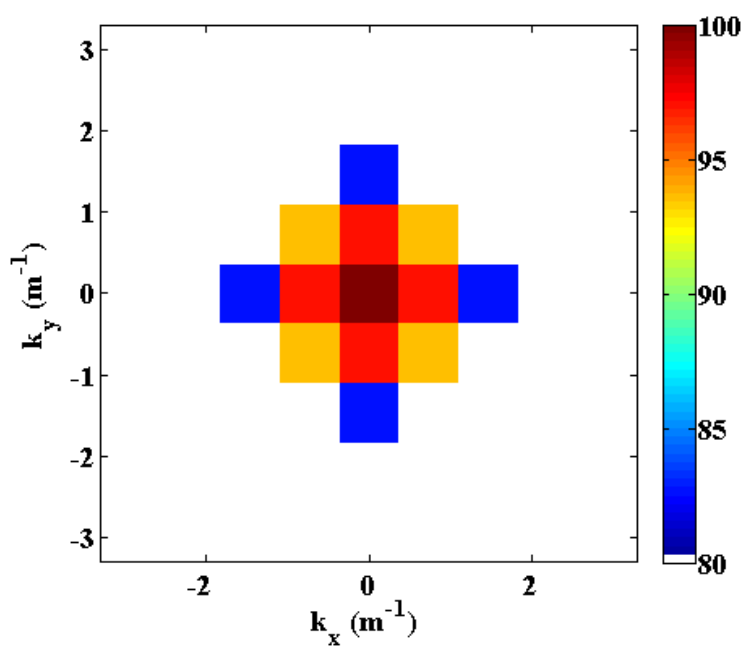

(a) Average autospectrum $\overline{\tilde{P}}\left(k_{x}, k_{y}, k_{x}, k_{y}\right)$

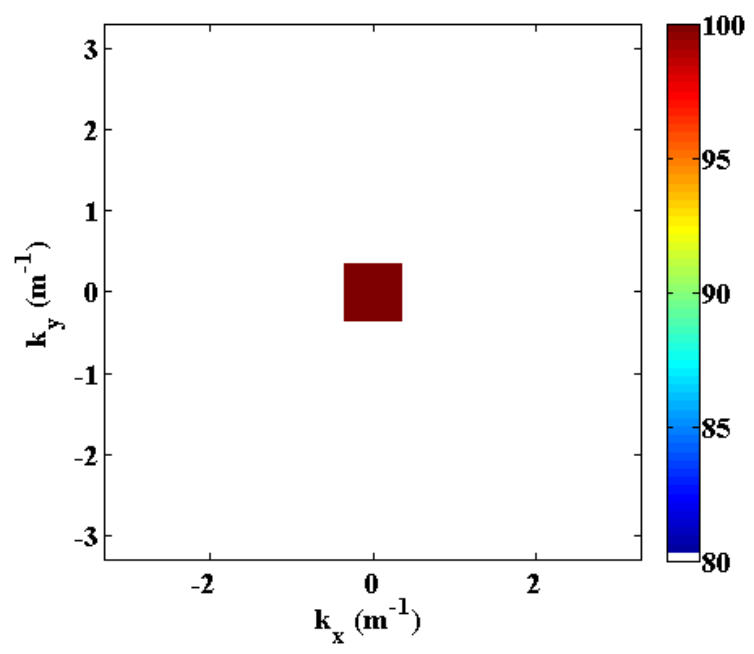

(c) Source autospectrum $\tilde{Q}\left(k_{x}, k_{y}, k_{x}, k_{y}\right)$

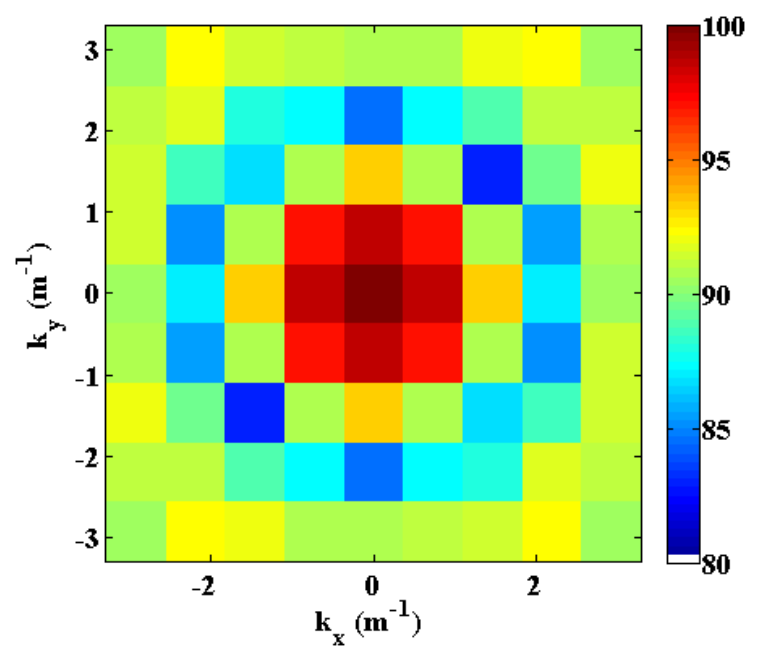

(b) Average cross-spectral magnitude $\left|\bar{P}\left(0,0, k_{x}, k_{y}\right)\right|$

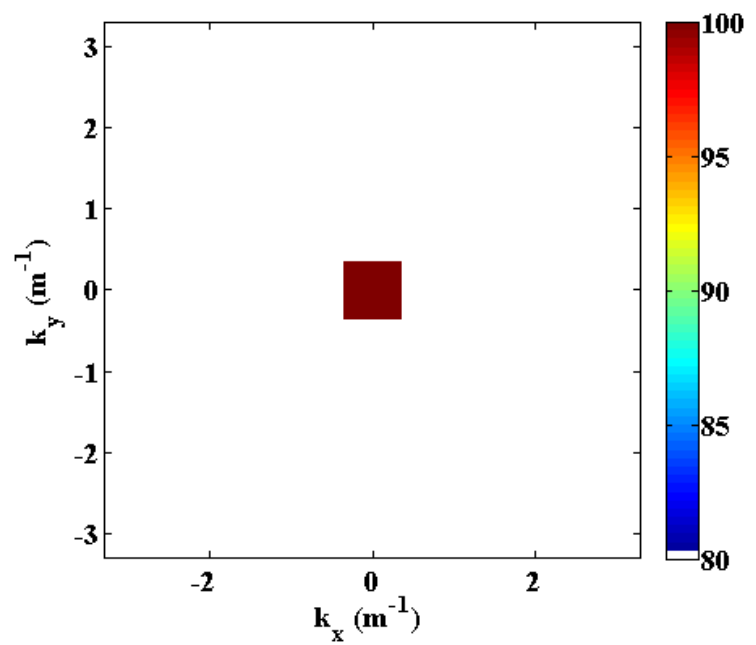

(d) Source cross-spectral magnitude $\left|\tilde{Q}\left(0,0, k_{x}, k_{y}\right)\right|$

Figure 3. Broadside plane wave pressure and source spectra.

\section{Coherent Plane Wave Pair}

A two source configuration is now analyzed. Here, a field of two plane waves arriving from opposite directions is simulated. The waves are defined to correspond to $k_{x}= \pm 1.46 \mathrm{~m}^{-1}, k_{y}=0 \mathrm{~m}^{-1}$. These values are selected to co-locate with grid-point centers. The plane waves are defined as being perfectly coherent, with the $k_{x}=1.46 \mathrm{~m}^{-1}$ wave having a $\pi / 2$ radian phase lag behind the $k_{x}=-1.46 \mathrm{~m}^{-1}$ wave. Wave magnitudes are set to $100 \mathrm{~dB}$. The average wavespace auto- and cross-spectra are respectively plotted in Figures $4 \mathrm{a}$ and $4 \mathrm{~b}$. The coordinates of the reference point for the cross-spectrum are selected to show the relationship of the first wave to the second. 
Convergence is reached after 150,000 iterations, showing a dramatic increase over the single point source case. Computation time scales similarly. The resultant source auto- and cross-spectra are shown in Figures 4c and 4d The source plots again behave as expected for perfectly-coherent sources, where the cross-spectral magnitude matches the autospectral magnitudes for equal-power sources. The phase angle between the two sources $\angle \tilde{Q}(-1.46,0,1.46,0)$ matches $\pi / 2$ to the third decimal place with a value of 1.5709 radians, or a relative error of $8.7 \times 10^{-5}$.

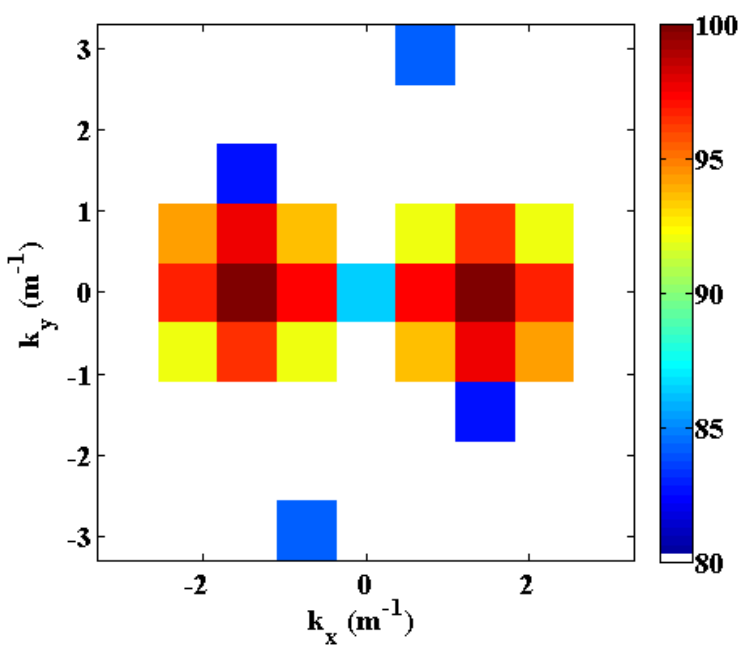

(a) Average autospectrum $\overline{\tilde{P}}\left(k_{x}, k_{y}, k_{x}, k_{y}\right)$

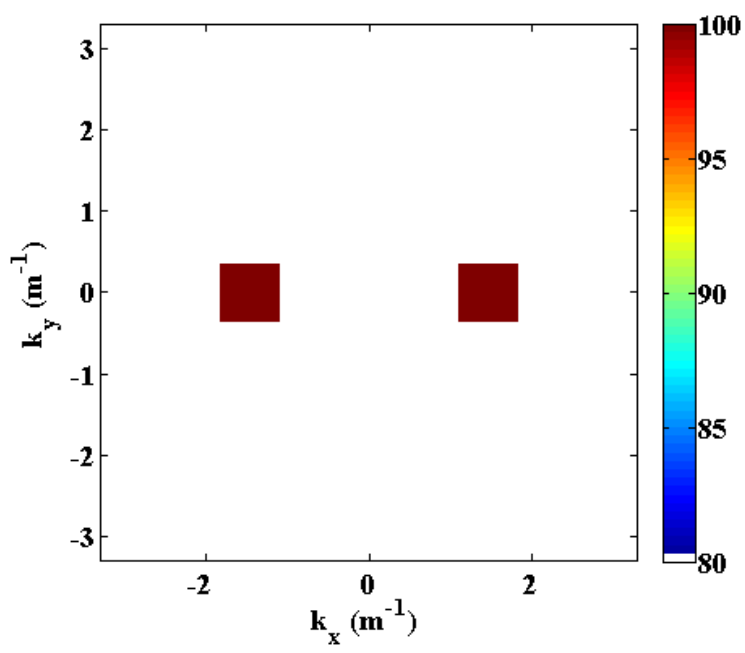

(c) Source autospectrum $\tilde{Q}\left(k_{x}, k_{y}, k_{x}, k_{y}\right)$

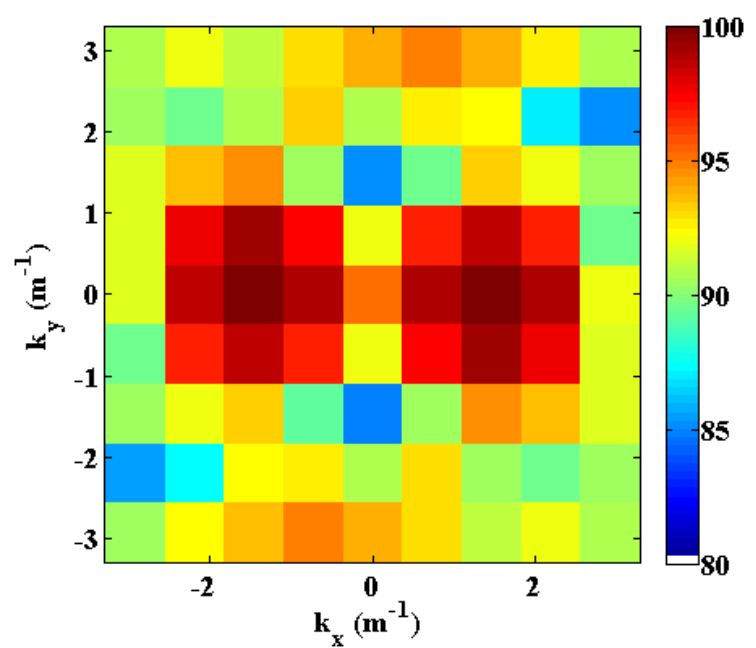

(b) Average cross-spectral magnitude $\left|\overline{\tilde{P}}\left(-1.46,0, k_{x}, k_{y}\right)\right|$

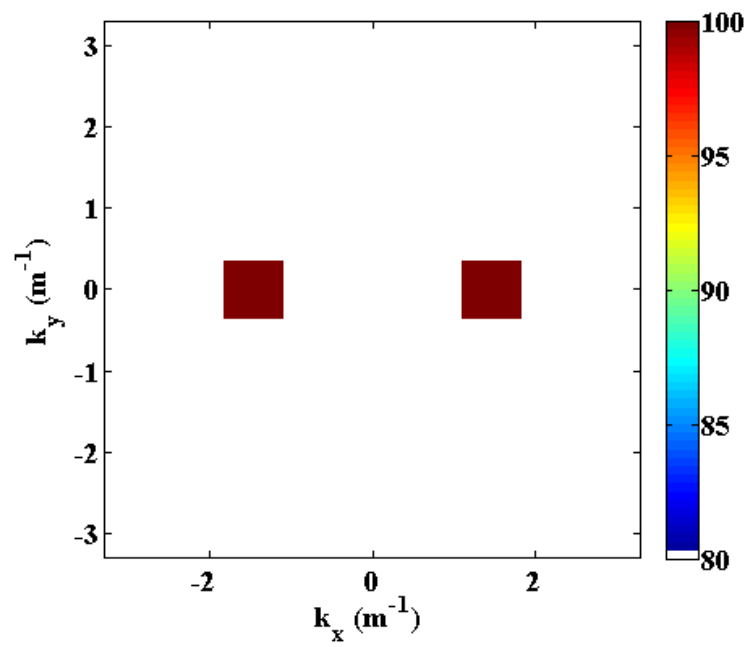

(d) Source cross-spectral magnitude $\left|\tilde{Q}\left(-1.46,0, k_{x}, k_{y}\right)\right|$

Figure 4. Coherent plane wave pair pressure and source spectra, $n_{k}=9$.

\section{Multiscale}

This solution is upscaled to an $n_{k}=13$ point grid ( 4 points per beamwidth) for the same wavespace extent $k_{\max }=2.92 \mathrm{~m}^{-1}$ and benchmarked. 1.5 million iterations are required for convergence at this scale. The upscaling method is attempted again with a reduction parameter of $\left(\Delta k_{n_{k}=13}\right)^{2} /\left(\Delta k_{n_{k}=9}\right)^{2}$. Since $\tilde{Q}$ is a 4 -D space of 2-D power spectra, this is equivalent to scaling power-spectral densities. This method fares no better with regard to numeric convergence, but does show correct results visually in fewer iterations.

Conversely, when the solver is started from a $\tilde{Q}=0$ condition for the same grid, only 500,000 iterations are required for convergence. This type of behavior is observed for most of the discrete, ideal plane wave cases considered. Based on observation of intermediate solution states, upscaling appears to bring the solution close to the correct value far more quickly than a zero-condition start. However, the solver takes a very long 
time to completely remove interpolation artifacts, so numerical convergence requires more iterations than the zero-start condition. Upscaling by the scaled solution reduces the influence of the interpolation artifacts, but shifts the initial guess of the correct bin further from the solution. This is sensible, as interpolating these single-wavenumber spatial tones to a more refined grid can be considered analogous to interpolating a temporal tone to a more refined spectrum with power-spectral density scaling. The level shift from the scaling causes a significant offset in the tone level. Upscaling is thus not considered for the remaining ideal plane wave simulations, as these are all defined as discrete spatial tones. However, power-spectral density-based upscaling is revisited in Section VI, where distributed field behavior is evident.

The 500,000 iteration zero-start case on the $n_{k}=13$ grid required just under 3 hours of run-time. The average pressure auto- and cross-spectra are respectively plotted in Figures $5 \mathrm{a}$ and $5 \mathrm{~b}$, The resultant source auto- and cross-spectra are shown in Figures $5 \mathrm{c}$ and $5 \mathrm{~d}$. The phase angle between the two sources $\angle \tilde{Q}(-1.46,0,1.46,0)$ shows slightly worse agreement with the true input, as it has a value of 1.5697 radians, or a relative error of $7.0 \times 10^{-4}$.

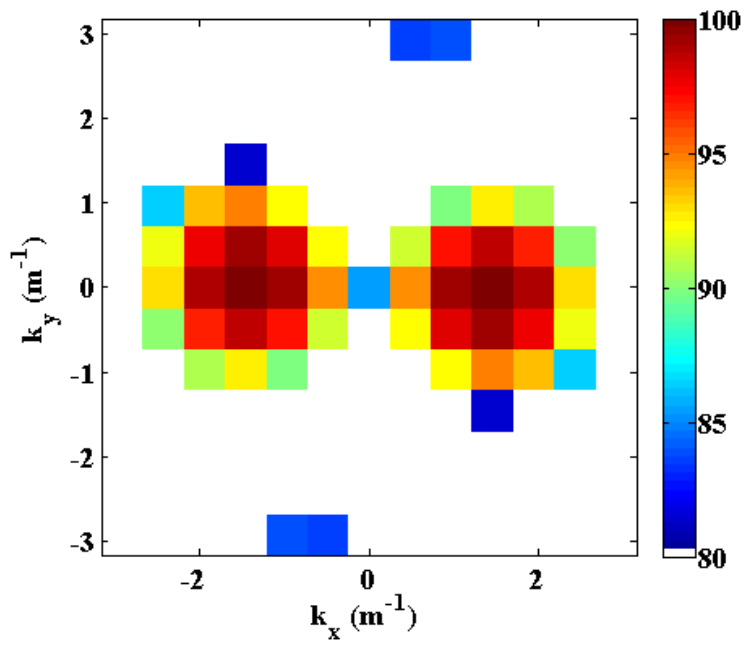

(a) Average autospectrum $\overline{\tilde{P}}\left(k_{x}, k_{y}, k_{x}, k_{y}\right)$

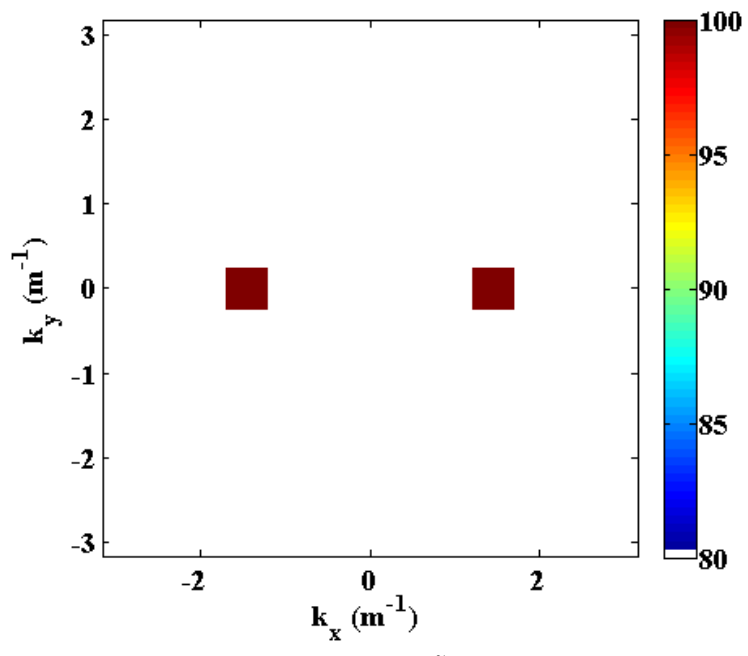

(c) Source autospectrum $\tilde{Q}\left(k_{x}, k_{y}, k_{x}, k_{y}\right)$

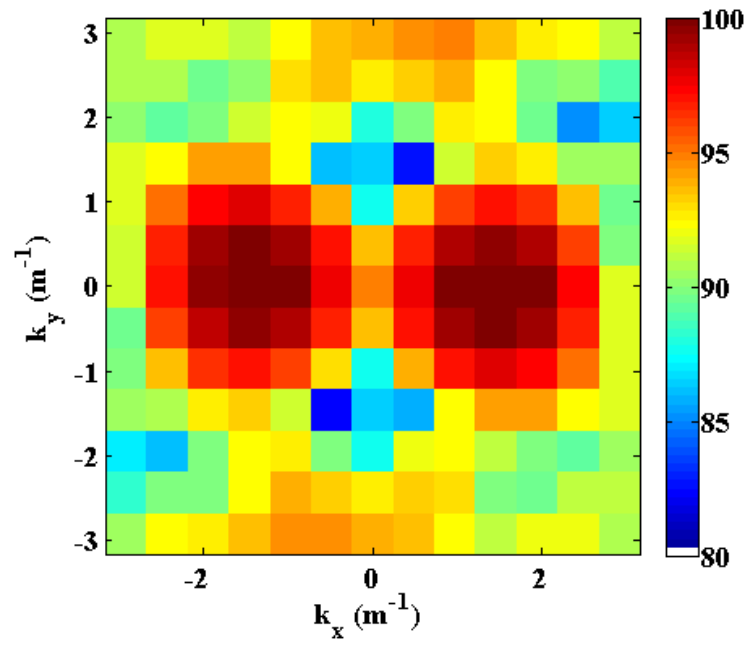

(b) Average cross-spectral magnitude $\left|\overline{\tilde{P}}\left(-1.46,0, k_{x}, k_{y}\right)\right|$

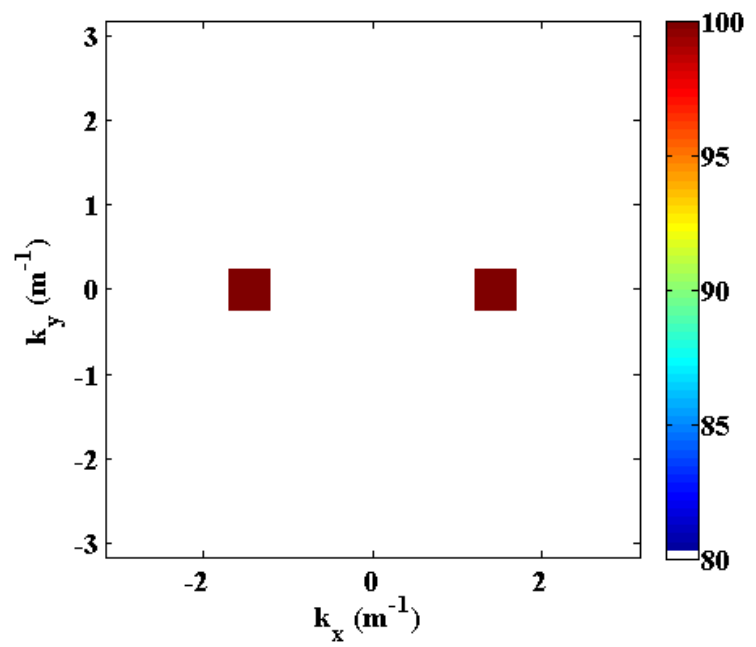

(d) Source cross-spectral magnitude $\left|\tilde{Q}\left(-1.46,0, k_{x}, k_{y}\right)\right|$

Figure 5. Coherent plane wave pair pressure and source spectra, $n_{k}=13$.

\section{Mixed-Coherence Isolated Plane Waves}

On the $n_{k}=9$ point grid, this two source configuration is further complicated with the addition of two incoherent sources to the field. The new plane waves again arrive from opposite directions, this time defined 
at $k_{x}=0 \mathrm{~m}^{-1}, k_{y}= \pm 1.46 \mathrm{~m}^{-1}$. The average auto- and cross-spectra are plotted in Figures $6 \mathrm{a}$ and $6 \mathrm{~b}$ The resultant source auto- and cross-spectra are shown in Figures $6 \mathrm{c}$ and $6 \mathrm{~d}$. Numeric convergence requires 500,000 iterations. As shown, the method properly separates the coherent and incoherent sources in the field. The phase angle $\angle \tilde{Q}(-1.46,0,1.46,0)$ is again matched to the third decimal place with a value of 1.5706 radians, or a relative error of $1.2 \times 10^{-4}$.

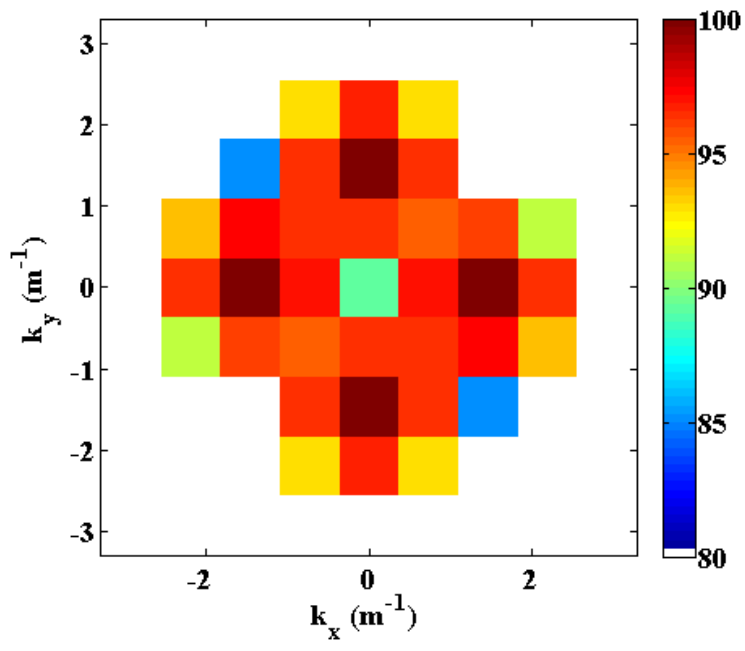

(a) Average autospectrum $\overline{\tilde{P}}\left(k_{x}, k_{y}, k_{x}, k_{y}\right)$

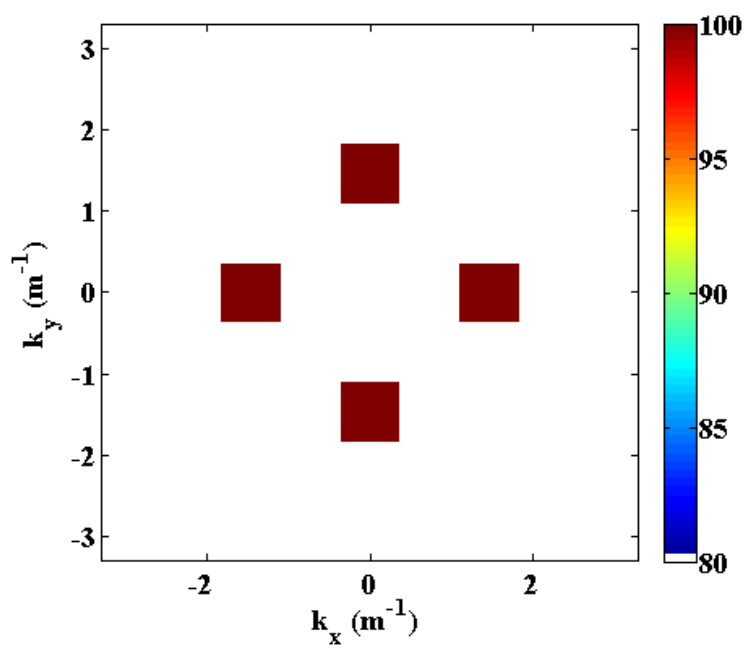

(c) Source autospectrum $\tilde{Q}\left(k_{x}, k_{y}, k_{x}, k_{y}\right)$

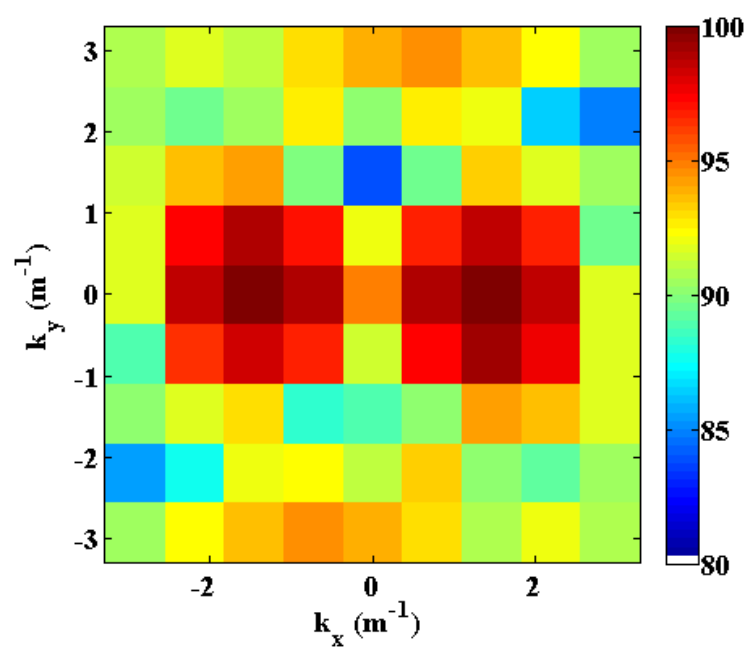

(b) Average cross-spectral magnitude $\left|\overline{\tilde{P}}\left(-1.46,0, k_{x}, k_{y}\right)\right|$

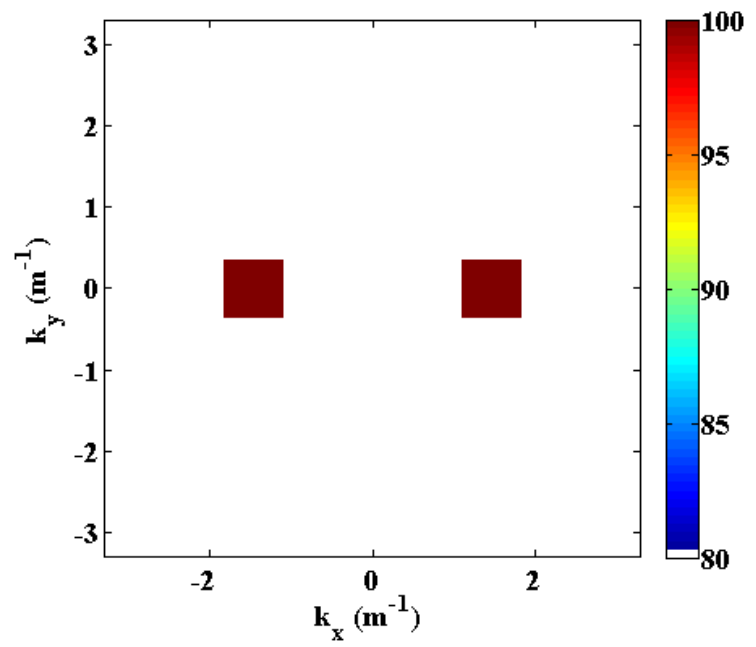

(d) Source cross-spectral magnitude $\left|\tilde{Q}\left(-1.46,0, k_{x}, k_{y}\right)\right|$

Figure 6. Pressure and source spectra for pair of coherent and pair of incoherent sources.

\section{Line Source with Decaying Coherence}

The final ideal plane wave case considered consists of a line of sources located along the $k_{x}$ axis, at values of $k_{x}=-1.46,-0.73,0,0.73,1.46 \mathrm{~m}^{-1}$ and $k_{y}=0 \mathrm{~m}^{-1}$. As before, all of the source auto-powers are set to 100 $\mathrm{dB}$. However, the sources are defined to have decaying coherence relative to the center of the line. Defining a coherence-squared function as

$$
\gamma^{2}\left(k_{\xi}, k_{\eta}, k_{x}, k_{y}\right)=\frac{\left|\tilde{Q}\left(k_{\xi}, k_{\eta}, k_{x}, k_{y}\right)\right|^{2}}{\tilde{Q}\left(k_{\xi}, k_{\eta}, k_{\xi}, k_{\eta}\right) \tilde{Q}\left(k_{x}, k_{y}, k_{x}, k_{y}\right)},
$$

the values referenced to the center are set to $\gamma^{2}(0,0, \pm 0.73,0)=0.5$ and $\gamma^{2}(0,0, \pm 1.46,0)=0.25$. The phase angles of the coherent powers relative to the center source are set to $\angle \tilde{Q}(0,0, \pm 0.73,0)=\mp \pi / 4$ radians and 
$\angle \tilde{Q}(0,0, \pm 1.46,0)=\mp \pi / 2$ radians.

The average pressure autospectrum is plotted in Figure $7 \mathrm{a}$. The average pressure cross-spectral magnitude is plotted in Figure $7 \mathrm{~b}$, A strong interaction between the sources is present, as the peak value of the pressure field is $6 \mathrm{~dB}$ higher than any of the input sources. This interaction is evident in both the auto- and crossspectra.

The algorithm is run for 5 million iterations and numerical convergence is not achieved, although residual levels are close to the convergence value. There is clearly a very strong convergence dependence on source field complexity. However, the results after 5 million iterations, plotted for the source field autospectrum in Figure $7 \mathrm{c}$ and cross-spectral magnitude in Figure $7 \mathrm{~d}$, show the expected source behavior, aside from one spurious source $20 \mathrm{~dB}$ below peak in the cross-spectrum. All of the autospectral values are at $100 \mathrm{~dB}$, and the cross-spectral values decay from $100 \mathrm{~dB}$ at $k_{x}=0 \mathrm{~m}^{-1}$ to $97 \mathrm{~dB}$ at $k_{x}= \pm 1.46 \mathrm{~m}^{-1}$.

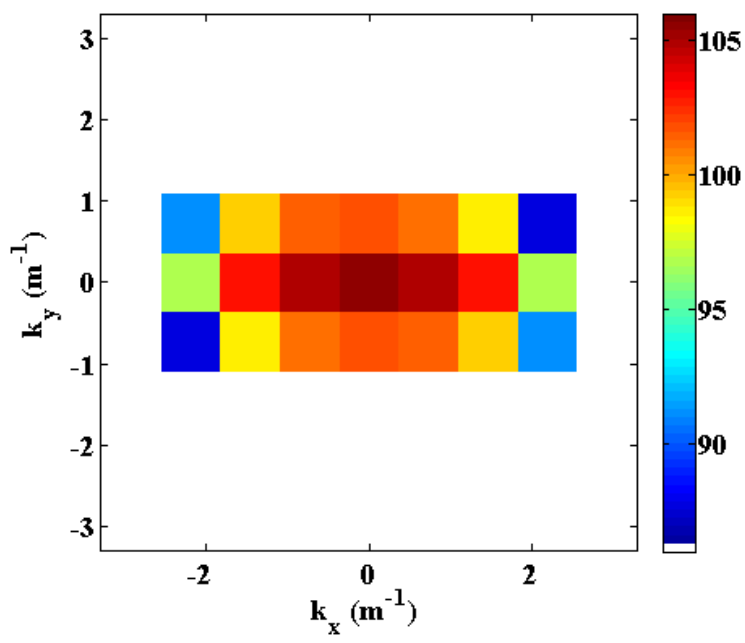

(a) Average autospectrum $\tilde{\tilde{P}}\left(k_{x}, k_{y}, k_{x}, k_{y}\right)$

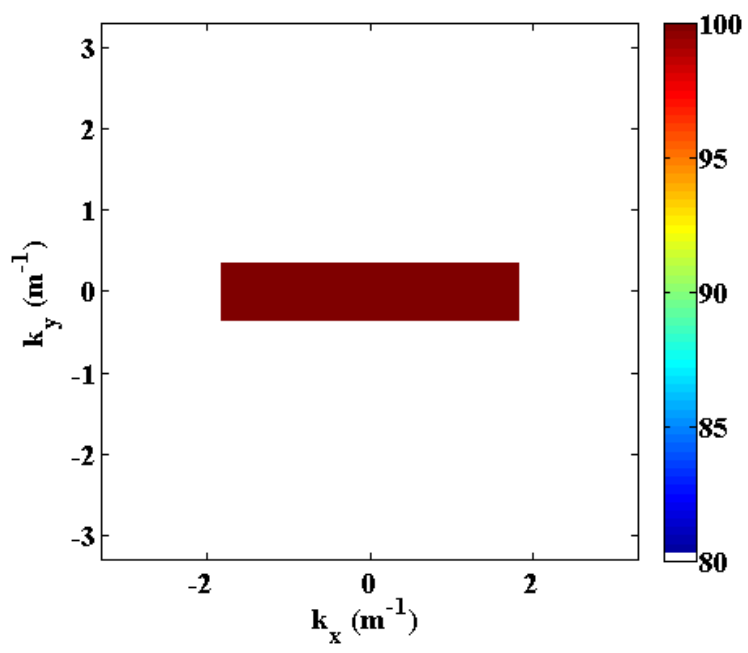

(c) Source autospectrum $\tilde{Q}\left(k_{x}, k_{y}, k_{x}, k_{y}\right)$

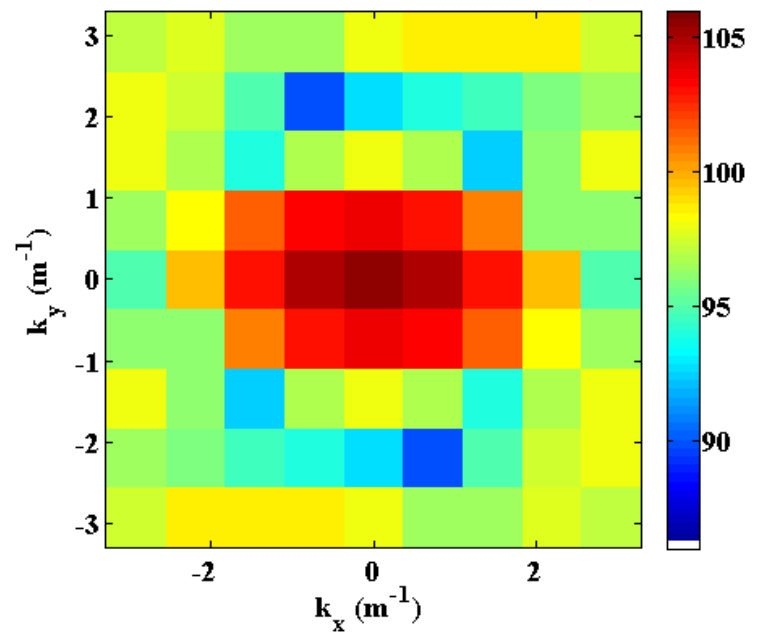

(b) Average cross-spectral magnitude $\left|\overline{\tilde{P}}\left(0,0, k_{x}, k_{y}\right)\right|$

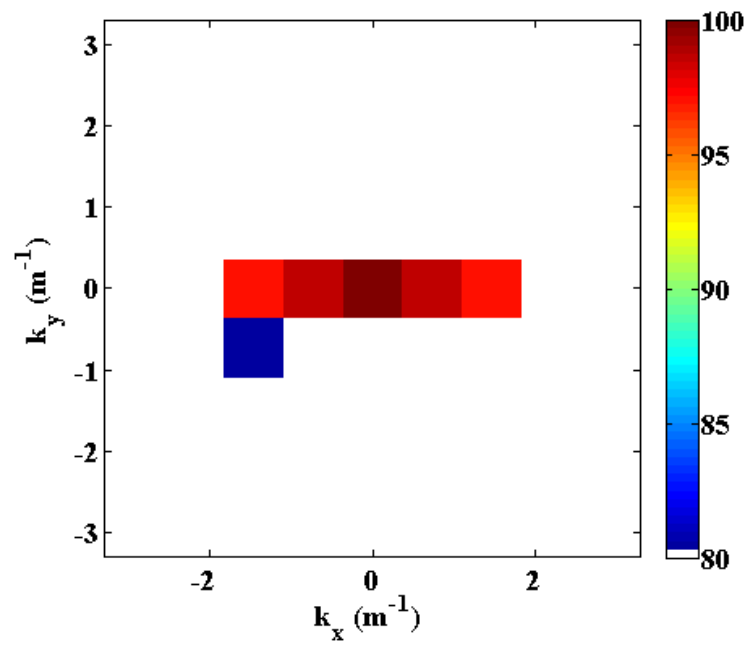

(d) Source cross-spectral magnitude $\left|\tilde{Q}\left(0,0, k_{x}, k_{y}\right)\right|$

Figure 7. Pressure and source spectra for a line of partially-coherent sources.

Figure 8 shows the behavior of the line of sources in more detail. Figure 8 a plots the cross-spectral magnitude $\left|\tilde{Q}\left(k_{\xi}, 0, k_{x}, 0\right)\right|$, as all of the non-zero elements of the true solution exist on this plane. As expected, the magnitude decays away from the autospectrum, which exists on the diagonal of the grid. Figure $8 \mathrm{~b}$ takes the $k_{\xi}=0$ cut of this plane and compares it to the true solution. As shown on a decibel scale, the results are close. Figure $8 \mathrm{c}$ then shows the cross-spectrum normalized to the coherence-squared and compares to the input values. Finally, Figure $8 \mathrm{~d}$ shows the resultant phase angles $\angle \tilde{Q}\left(0,0, k_{x}, 0\right)$ compared 
to the input angles. From this simulation, it is apparent that true numerical convergence is not necessary for reasonable results. However, not shown are the partial results from the first 2 million iterations, where spectral levels were still over $1 \mathrm{~dB}$ off, and phase angle errors were greater than $5 \%$.

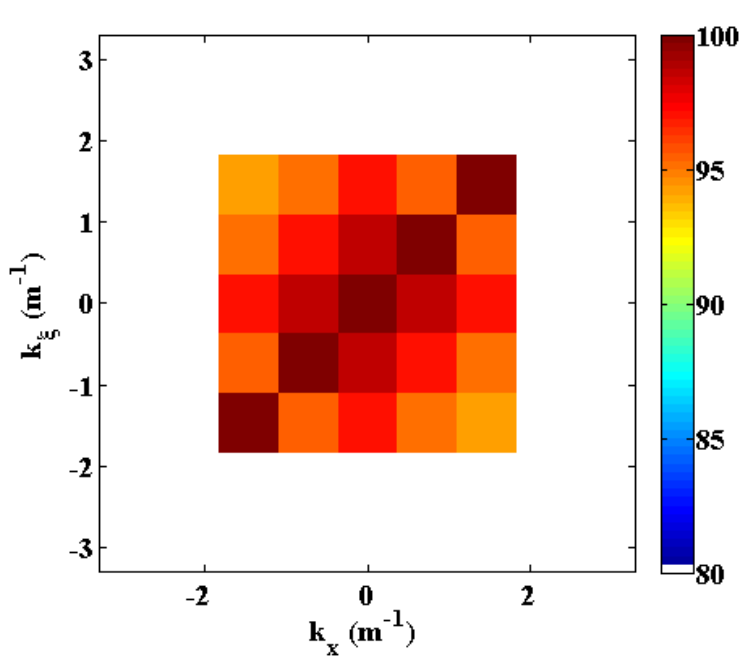

(a) $k_{\eta}=k_{y}=0$ plane, $\left|\tilde{Q}\left(k_{\xi}, 0, k_{x}, 0\right)\right|$

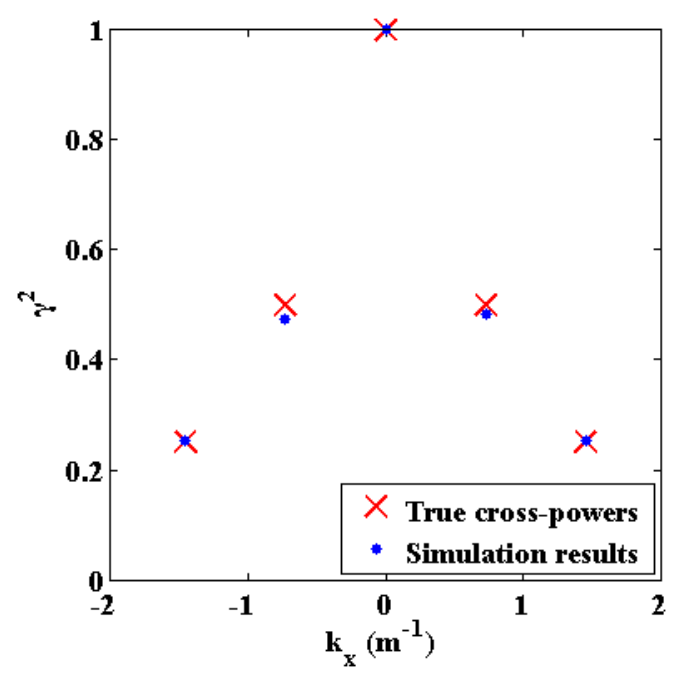

(c) Coherence-squared $\gamma^{2}\left(0,0, k_{x}, 0\right)$

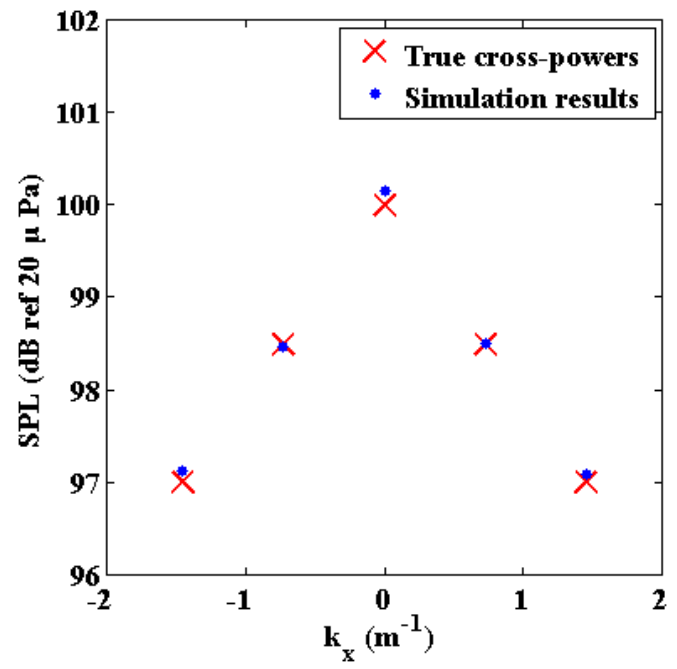

(b) Cross-spectral magnitude $\left|\tilde{Q}\left(0,0, k_{x}, 0\right)\right|$

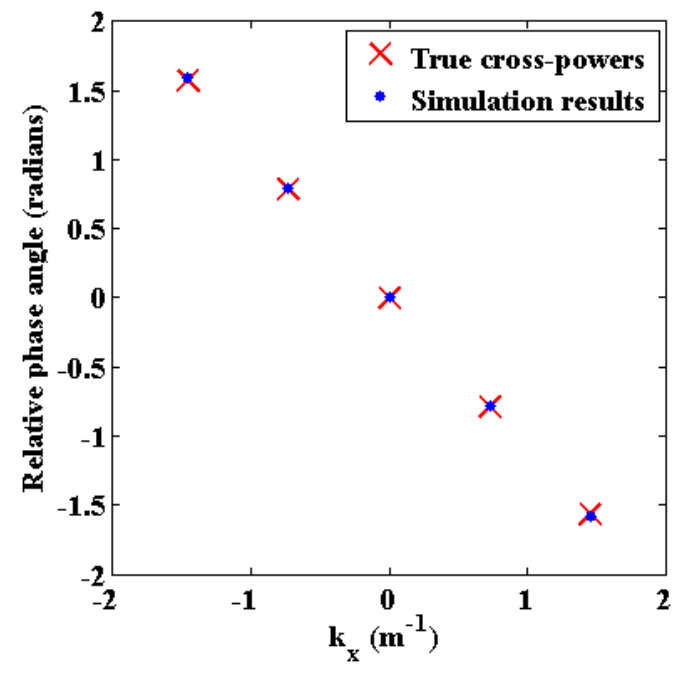

(d) Relative phase angles $\angle \tilde{Q}\left(0,0, k_{x}, 0\right)$

Figure 8. Evaluation of line source results and comparison to inputs.

\section{Curved-Wave Beamforming}

Results from Section V show that this deconvolution formulation is functional, if expensive, for ideal discrete plane waves of varying coherence. However, under many circumstances an aeroacoustic array will observe an acoustic field which consists of many curved wave fronts. While a true curved wave front cannot be fully represented by a select few frequencies in wavespace, it may be possible to find a "best-fit" wavespace spectrum to a given curved wave field.

\section{Simulated Results}

An initial simulation consists of a point source, centered over the array, located $1.5 \mathrm{~m}$ away from the array center, for a source coordinate of $(0,0,1.5)$. This location is selected as approximately twice the array aperture to generate a moderately-curved wavefront as observed by the acoustic array. The source is scaled such that 
its acoustic field has a level of $90.5 \mathrm{~dB}$ at the array center, and $90.2 \mathrm{~dB}$ at the outer microphone ring. As the acoustic field of this point source will appear as the superposition of many plane waves, the wavespace spectrum must be constructed to capture the entire acoustic radiation circle. If $k_{\max }$ from the previous sections is maintained and set to the radius of the radiation circle, computed as $k_{\max }=f / c_{0} \frac{25}{2}$ this sets the source frequency at $1 \mathrm{kHz}$. The average spectrum $\bar{P}$ is constructed on three grid scales of $n_{k}=9, n_{k}=13$ and $n_{k}=17$. For constant $k_{\max }$, this gives successively smaller $\Delta k$ values, corresponding to increasingly large effective array apertures. Numeric convergence is not expected for these cases based on previous results, so the algorithm is run until residuals and error no longer decrease. Figure 9a shows the average pressure autospectrum $\tilde{P}$ for the $n_{k}=9$ grid, while Figure $9 \mathrm{~b}$ shows the solution autospectrum after 500,000 iterations. This solution is then upscaled to the $n_{k}=13$ grid. The $n_{k}=13$ average pressure autospectrum and solution autospectrum after 50,000 iterations are shown in Figures 9c and 9d. This solution is then upscaled to the $n_{k}=17$ grid, with average pressure spectrum and solution after 20,000 iterations shown in Figures $9 \mathrm{e}$ and $9 \mathrm{f}$.

The plotted wavespace results appear as a cone of arrival directions centered about the broadside direction, as expected. For this simulation, the array occupies a $28^{\circ}$ solid angle of the source's radiation pattern. While explicit definition of the true solution for a spatially-varying, array-sampled, curved wavefront in wavespace may be difficult, it would not be unreasonable to expect that the source field have the majority of its power occupy a direction-of-arrival cone corresponding to this solid angle. For this case, the energy should reside approximately within a circle of radius $k=0.7 \mathrm{~m}^{-1}$, which is seen for all three $n_{k}$ values. Quantitatively, the total summed power within the source autospectra is $89.5 \mathrm{~dB}$ for $n_{k}=9,88.9 \mathrm{~dB}$ for $n_{k}=13$, and 88.4 $\mathrm{dB}$ for $n_{k}=17$. These values do not match the expected levels for the acoustic power observed by the array. However, if the total source field is summed over all four wavespace dimensions, the total coherent power sum can be computed. This total summed power within the wavespace spectrum is $90.5 \mathrm{~dB}$ for all three grid densities, matching the true acoustic field power at the array center. The $n_{k}=17$ grid is restarted with a zero-source field to evaluate the effects of upscaling. This case is run for 20,000 iterations. For brevity, no plots are shown. The total summed power of the source field for this case is still $90.5 \mathrm{~dB}$. However, the summed autospectral power is only $80.9 \mathrm{~dB}$. While there is no true solution to compare and say which result is closer, the error as defined in Equation $(19)$ is two orders of magnitude smaller for the upscaled case than for the zero-start case.

\section{Experimental Results: Trailing Edge Noise}

The final case considered is taken from an existing trailing edge noise data set collected in UFAFF 26 Data are acquired for a $0.3048 \mathrm{~m}$ chord, $0.74 \mathrm{~m}$ span NACA 0012 airfoil, in this case at a Mach number of 0.17. Array data are acquired for an array with the same layout as that used in all previous simulations. A laser pulse calibration technique is applied to the data $C S M$ to calibrate the individual microphone channels and, to first order, correct for shear layer refraction effects. ${ }^{16}$ Additional details of the experiment can be found in the reference. A photograph of the installation is shown in Figure 10 while a legend of the illustrations used in the baseline beam map at $z=1.13 \mathrm{~m}$ is shown in Figure 11. Here, flow is from right to left. The airfoil trailing edge center span is located at $(0,0,1.13) \mathrm{m}$ from the array center. A conventional beam map of the array data is shown in Figure 12. Positive $x$ in the beam map points in the upstream direction. Positive $y$ points upward vertically, while positive $z$ points out of the page. The airfoil trailing edge appears as the dominant noise source.

The wavespace deconvolution procedure is applied to the $2 \mathrm{kHz}$ narrowband $C S M$ computed from the experiment. This is the only frequency bin considered for the following analysis. This increase in frequency from $1 \mathrm{kHz}$ to $2 \mathrm{kHz}$ requires that, to maintain beamwidth resolution, $n_{k}$ be increased to 33 points to account for the increase in acoustic wavenumber and $k_{\max }$.

The algorithm is run on a succession of grids of $n_{k}=9, n_{k}=13, n_{k}=17, n_{k}=25$ and $n_{k}=33$. A surprising effect is observed for this case. While in previous cases the algorithm required a large number of iterations at every stage, here a clean, logarithmic roll-off in the error and residual from Eqs. (19) and 20) is observed, and relatively few iterations are required for near-total single-precision convergence. At $n_{k}=9,10,000$ iterations are required. However, at both $n_{k}=13$ and $n_{k}=17$, only 3,000 are necessary. At $n_{k}=2515,000$ are required for convergence, and finally 20,000 are needed for $n_{k}=33$. This requires just over 7 hours of computation time. The validity of this upscaling is evaluated by running the $n_{k}=17$ grid from a zero-start. The autospectrum quickly attains a very similar shape to that seen in the upscaling 


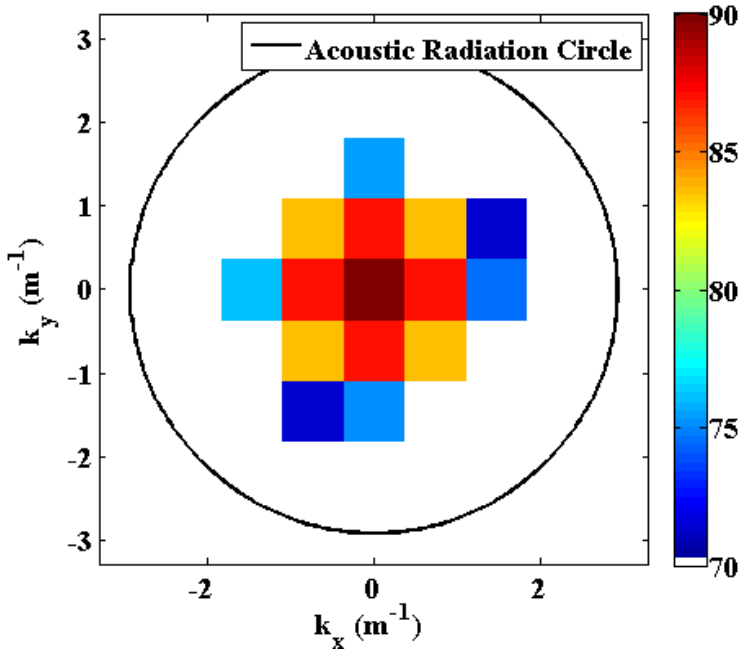

(a) $\bar{P}\left(k_{x}, k_{y}, k_{x}, k_{y}\right), n_{k}=9$

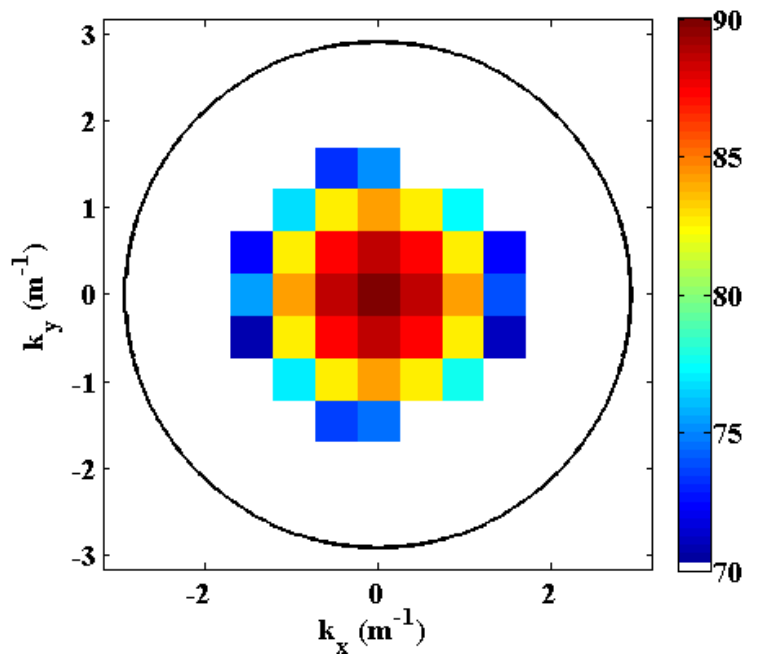

(c) $\tilde{P}\left(k_{x}, k_{y}, k_{x}, k_{y}\right), n_{k}=13$

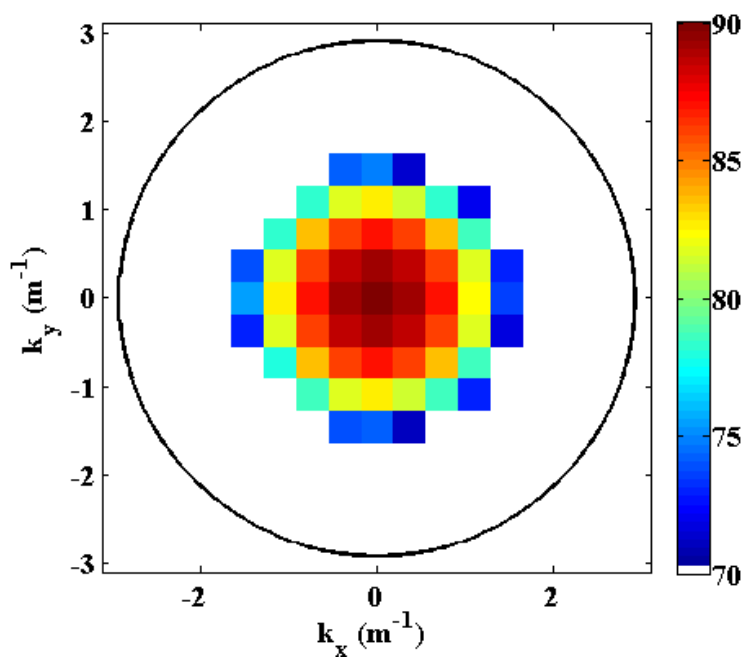

(e) $\bar{P}\left(k_{x}, k_{y}, k_{x}, k_{y}\right), n_{k}=17$

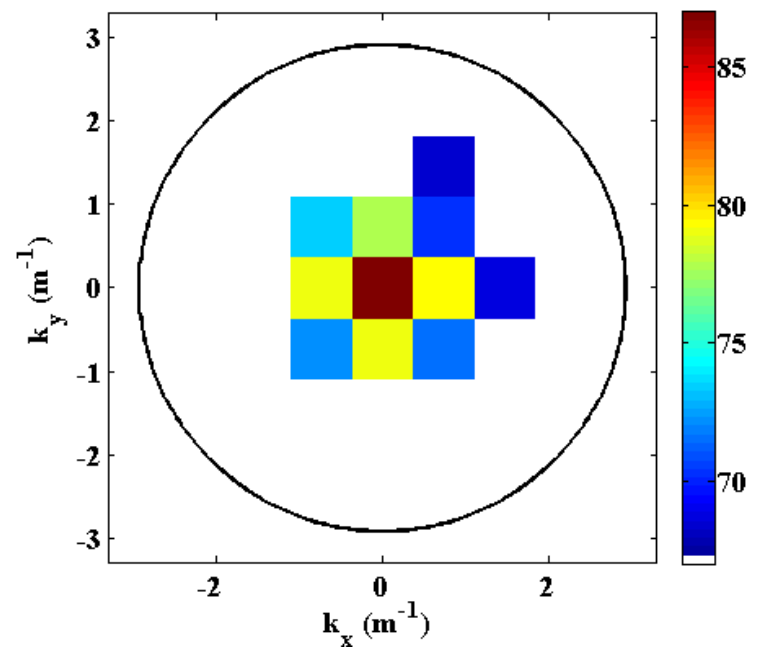

(b) $\tilde{Q}\left(k_{x}, k_{y}, k_{x}, k_{y}\right), n_{k}=9$

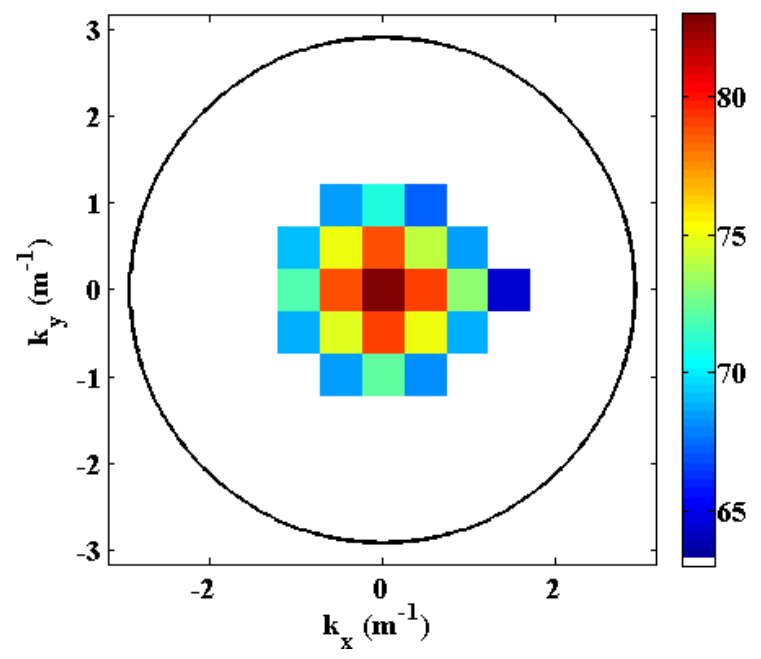

(d) $\tilde{Q}\left(k_{x}, k_{y}, k_{x}, k_{y}\right), n_{k}=13$

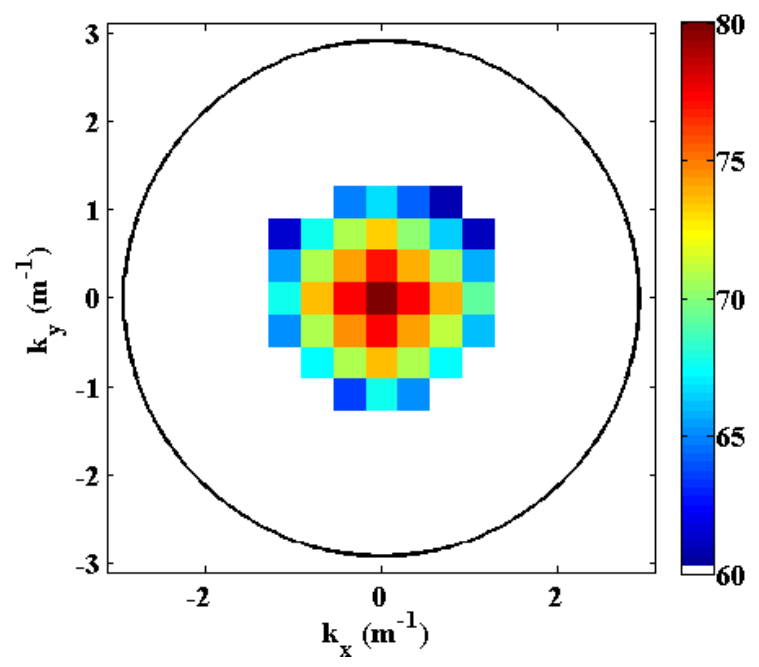

(f) $\tilde{Q}\left(k_{x}, k_{y}, k_{x}, k_{y}\right), n_{k}=17$

Figure 9. Pressure and source autospectra for a near-array point source. 


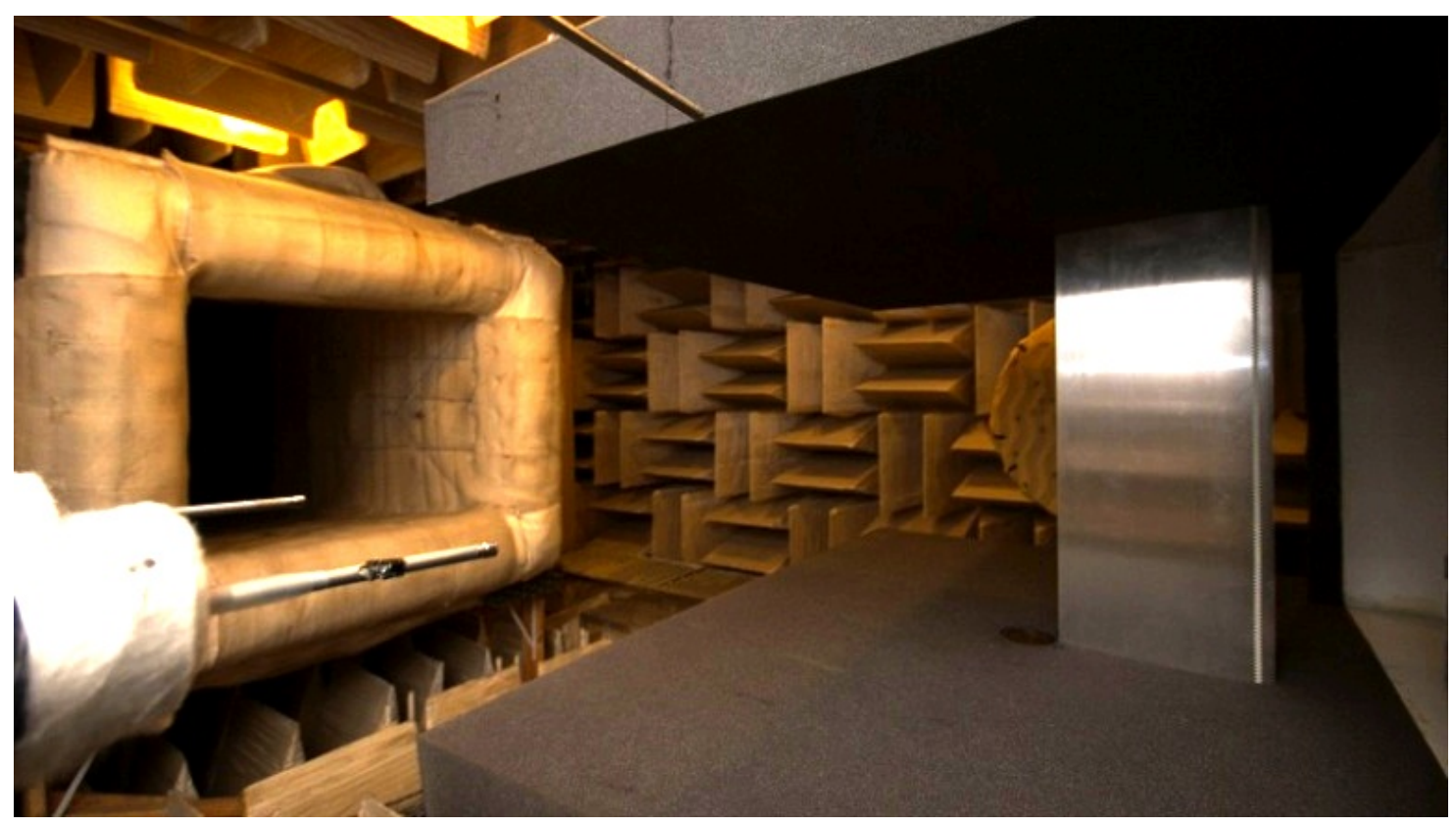

Figure 10. NACA 0012 trailing edge noise measurement installation.

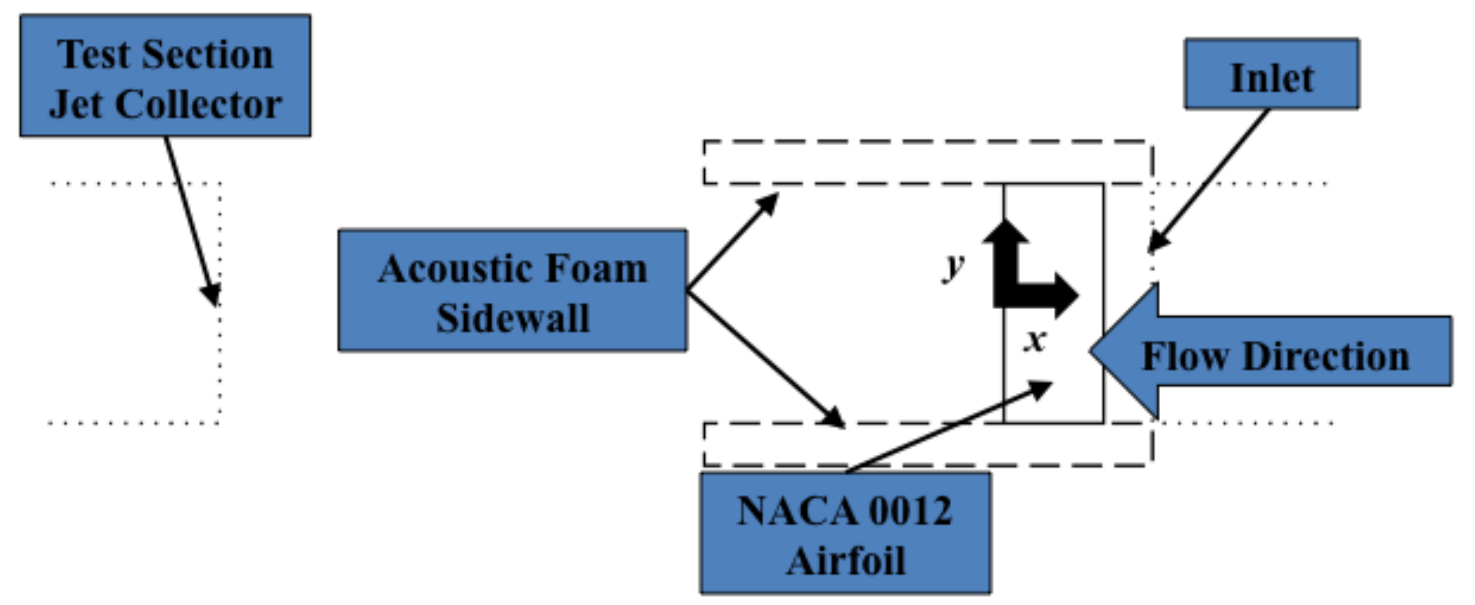

Figure 11. Facility boundary lines as drawn in beam map. 


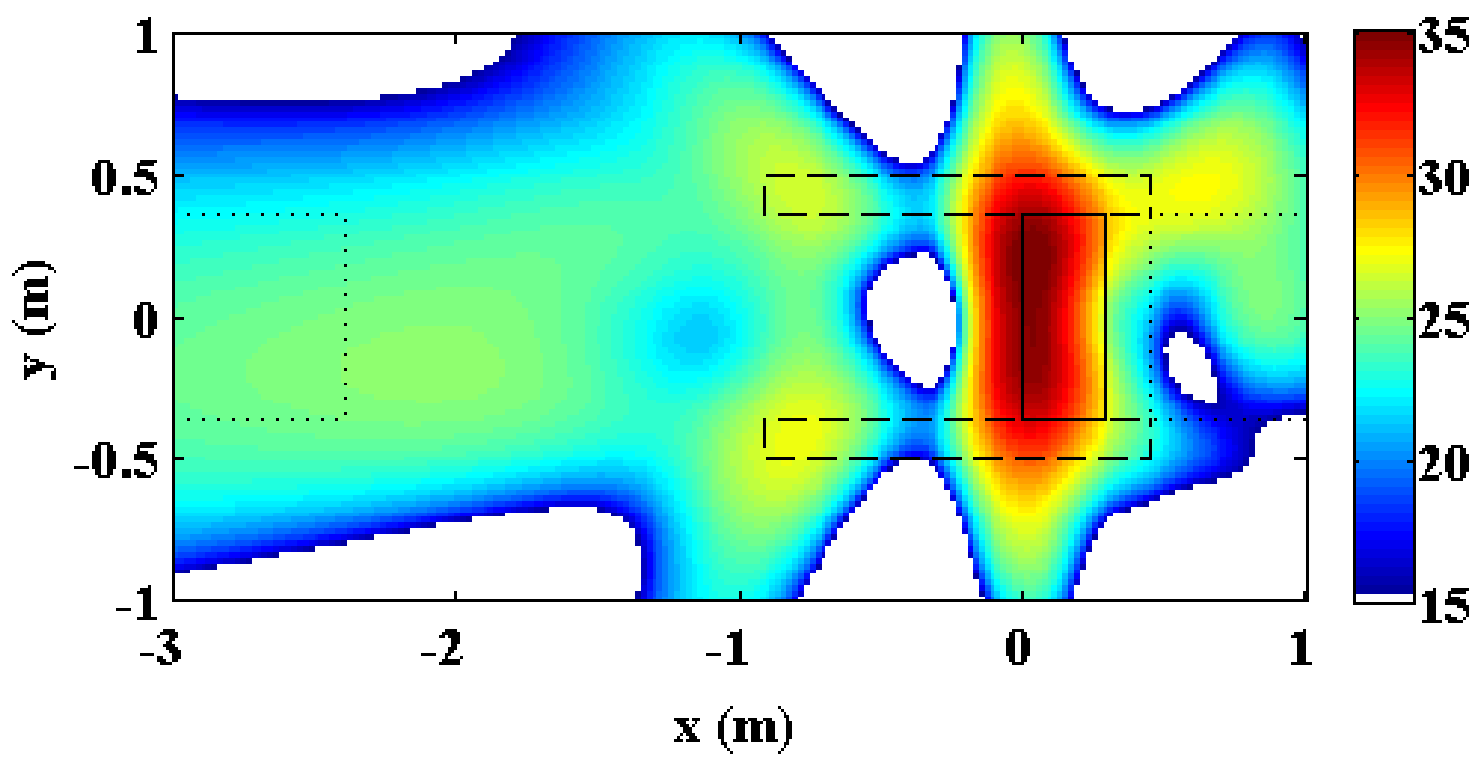

Figure 12. Baseline beamforming for NACA 0012 experiment, $z=1.13 \mathrm{~m}$.

process, but levels rise slowly and, after an initial drop, the error and residual behavior is worse. There are many possible reasons for this. The effect may indicate that the interpolation scheme is reasonable for this case, or that this particular wave field lends itself readily to representation by a summation of plane waves. Alternately, the sampling grid may have better conditioning, or there could be some other numerical effect such as floating point precision which did not matter for smaller grids, but comes into play here. Further investigation is necessary.

The resultant average and source autospectra for the $n_{k}=9, n_{k}=17$ and $n_{k}=33$ grids are shown in Figure 13. Broadside waves at $k_{x}=0$ are clearly present, as expected for an airfoil trailing edge acoustic source located at $x=0 \mathrm{~m}$. Additionally, waves arriving from downstream, located near $k_{x}=-4 \mathrm{~m}^{-1}$, are likely due to the facility diffuser. Additional low-level sources appear outside of the radiation circle. Based on the average pressure spectra, these may not be sensible. Additional comparison of upscaled and zero-start solutions is likely required. The total sum power of the wavespace spectrum for the trailing edge region of the source field for $n_{k}=33$ is $45.7 \mathrm{~dB}$. The sum power of the autospectrum in this region and at this scale is $37.6 \mathrm{~dB}$. The two-microphone method results from the reference, concluded to be the most accurate estimate of the trailing edge noise for this frequency, showed a trailing edge noise level of $48 \mathrm{~dB}$.

\section{Proposed Extension to Nearfield Beamforming}

Initial results for curved waves indicate that approximate source region powers may be computed by summing the appropriate subset of wavespace auto- and cross-powers for given directions-of-arrival of interest. However, interpretation of these results is, at the moment, difficult and potentially non-intuitive depending on source configuration. However, with a full cross-power spectrum in wavespace, a synthetic CSM may be constructed by inverse-transforming the spectrum $\tilde{Q}$. This $C S M$ can be used with conventional beamforming in an attempt to further isolate sources, and possibly with cross-beamforming ${ }^{7}$ to determine regional coherence. Initial tests with this process show qualitative success, but are far from quantitative reliability. Further investigation of this is left to future work.

\section{Summary and Future Work}

A coherent deconvolution technique is presented for a class of shift-invariant problems constructed in wavespace. The deconvolution method is based on an iterative solver which enforces appropriate constraints after every iteration. The solver iteratively evaluates a forward convolution utilizing a four-dimensional FFT, providing improved scaling with problem size when compared to matrix methods. The technique is applied to ideal plane wave cases successfully, and properly recovers both magnitude and phase of cross-spectral 


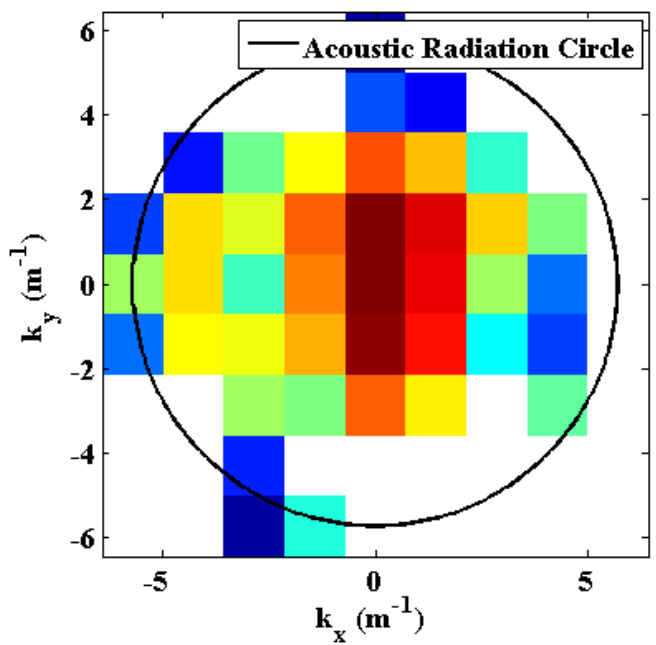

(a) $\overline{\tilde{P}}\left(k_{x}, k_{y}, k_{x}, k_{y}\right), n_{k}=9$

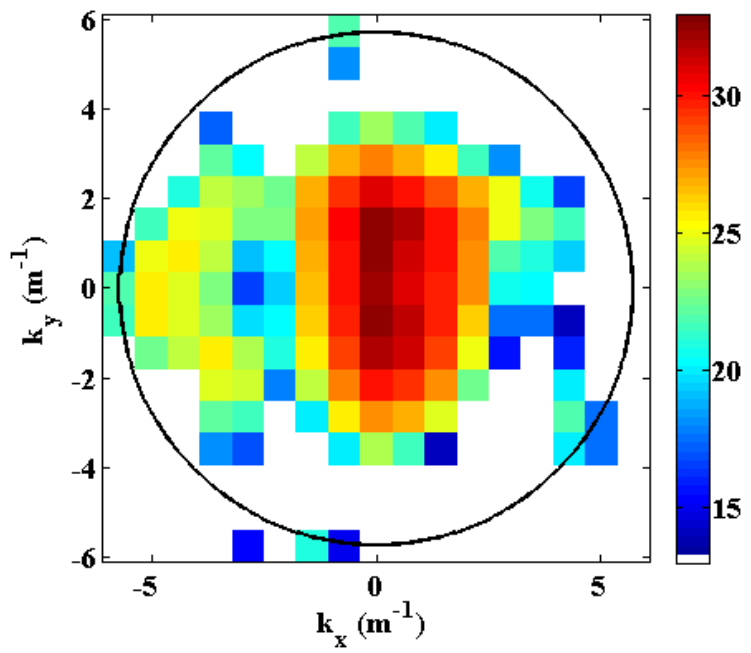

(c) $\overline{\tilde{P}}\left(k_{x}, k_{y}, k_{x}, k_{y}\right), n_{k}=17$

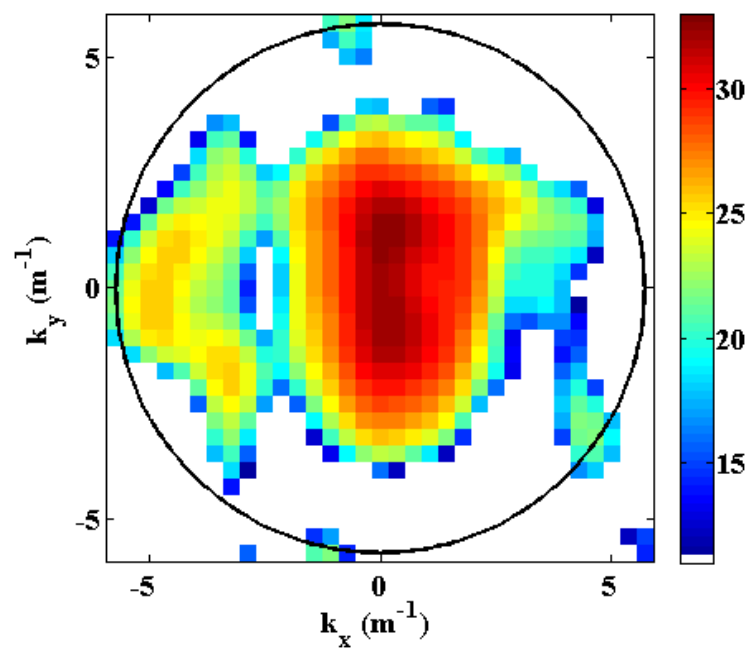

(e) $\overline{\tilde{P}}\left(k_{x}, k_{y}, k_{x}, k_{y}\right), n_{k}=33$

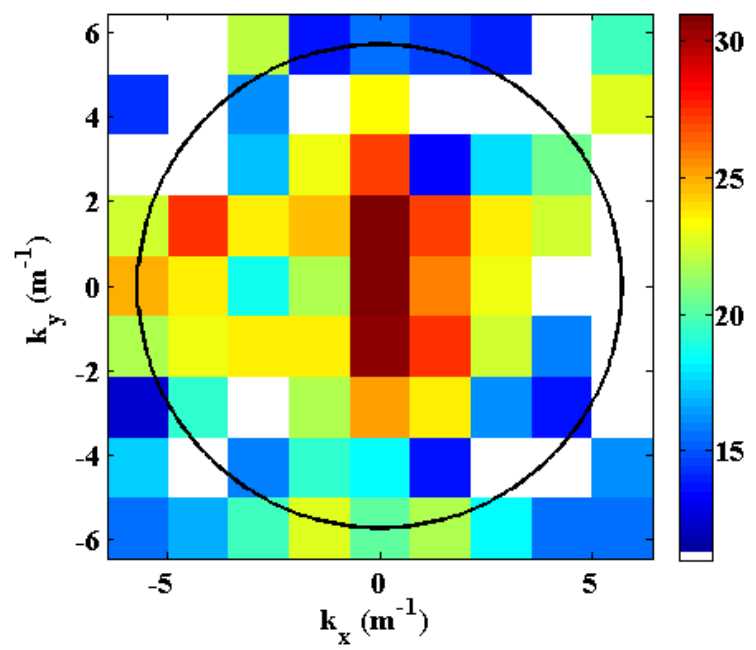

(b) $\tilde{Q}\left(k_{x}, k_{y}, k_{x}, k_{y}\right), n_{k}=9$

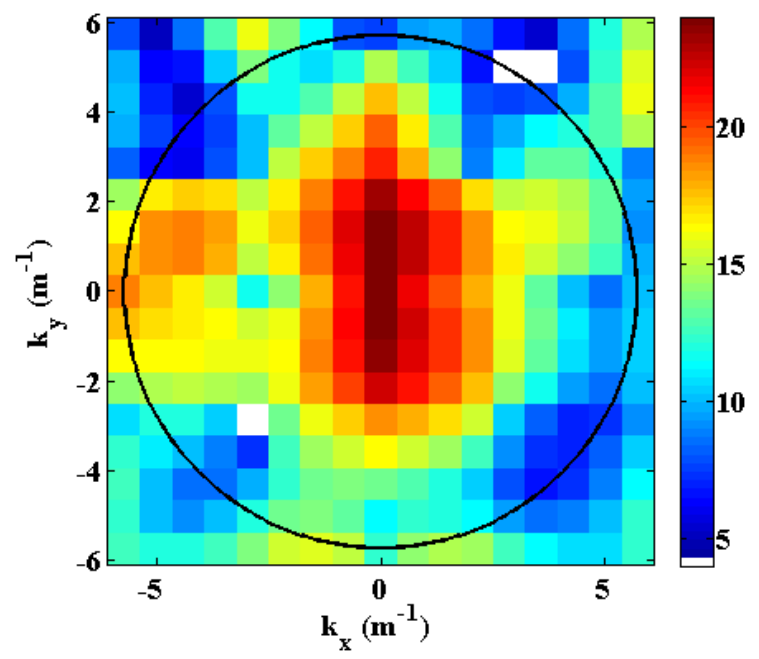

(d) $\tilde{Q}\left(k_{x}, k_{y}, k_{x}, k_{y}\right), n_{k}=17$

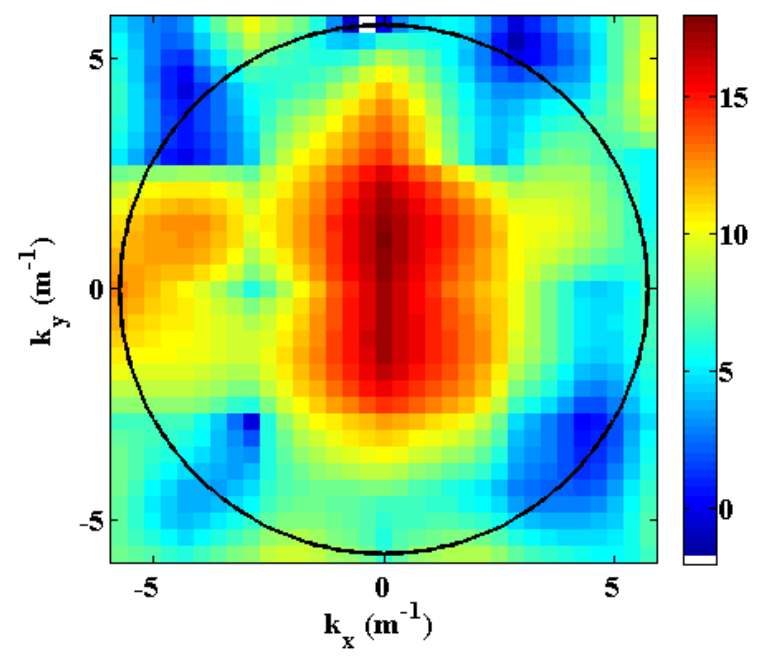

(f) $\tilde{Q}\left(k_{x}, k_{y}, k_{x}, k_{y}\right), n_{k}=33$

Figure 13. Pressure and source autospectra for the NACA 0012 experiment. 
relationships between distinct waves of arbitrary coherence. Acceleration through an improved calculation of a stable relaxation parameter greatly improves the behavior of the algorithm. Upscaling for an initial guess on a given grid performs poorly for discrete plane waves when combined with linear interpolation. However, it appears to provide an improved initial guess for true distributed noise sources.

A large amount of work remains to properly evaluate this method. First and foremost, more curved wave simulations must be conducted with a variety of multi-source configurations. The reconstruction of synthetic $C S M$ s must be pursued. The upscaling procedure must be further investigated with regard to the convergence behavior of the trailing edge noise data set. Additionally, the effect of the point source shear layer correction on the data is not considered here, and may affect solution behavior. Experimental data which do not require such correction should be analyzed in any future study. The effect of floating-point precision on larger grids with real data should be considered.

Acceleration must continue to be pursued. While FFTs show favorable scaling with advances in computational technology, alternative means of improving solution times must still be sought. Multigrid methods may prove to be the most promising for distributed noise fields, but four-dimensional interpolation of crossspectra must be evaluated in more detail, given the observed behavior with discrete plane waves. Careless application of multigrid or even simple upscaling may prove problematic if both distributed noise sources and discrete plane waves are present in a given acoustic field. Alternatively, other algorithms may be explored. The iterative technique considered here is one of many ways to pursue what amounts to a constrained optimization problem. Finding a technique which does not require tens or hundreds of thousands of iterations to reach a low-residual solution would be of huge benefit to this deconvolution method. Finally, while this wavespace deconvolution algorithm circumvents the full computation and storage of the coefficient matrix $\boldsymbol{A}$, the problem still shows poor scaling for high frequencies, especially with regards to memory consumption. An alternative method of performing the forward convolution which avoids the storage and computation associated with four-dimensional zero-padding could show large improvements in both computation time and memory requirements.

\section{References}

\footnotetext{
${ }^{1}$ Brooks, T. F. and Humphreys, W. M., "A deconvolution approach for the mapping of acoustic sources (DAMAS) determined from phased microphone arrays," Journal of Sound and Vibration, Vol. 294, 2006, pp. 856-879.

${ }^{2}$ Dougherty, R. P., "Extensions of DAMAS and Benefits and Limitations of Deconvolution in Beamforming," 11th AIAA/CEAS Aeroacoustics Conference, AIAA-2005-2961, Monterey, CA, May 2005.

${ }^{3}$ Yardibi, T., Li, J., Stoica, P., and Cattafesta, L., "Sparsity Constrained Deconvolution Approaches for Acoustic Source Mapping," Journal of the Acoustical Society of America, Vol. 123, No. 5, 2008, pp. 2631-2642.

${ }^{4}$ Sijtsma, P., "CLEAN Based on Spatial Source Coherence," International Journal of Aeroacoustics, Vol. 6, No. 4, 2007, pp. 357-374.

${ }^{5}$ Yardibi, T., Li, J., Stoica, P., Zawodny, N. S., and Cattafesta, L., "A Covariance-Fitting Approach for Correlated Acoustic Source Mapping," Journal of the Acoustical Society of America, Vol. 127, No. 5, 2010, pp. 2920-2931.

${ }^{6}$ Ravetta, P. A., Burdisso, R. A., and Ng, W. F., "Noise Source Localization and Optimization of Phased-Array Results," AIAA Journal, Vol. 47, No. 11, 2009, pp. 2520-2533.

${ }^{7}$ Brooks, T. F. and Humphreys, W. M., "Extension of DAMAS Phased Array Processing for Spatial Coherence Determination (DAMAS-C)," 12th AIAA/CEAS Aeroacoustics Conference, AIAA-2006-2654, Cambridge, MA, May 2006.

${ }^{8}$ Suzuki, T., " $L_{1}$ generalized inverse beam-forming algorithm resolving coherent/incoherent, distributed and multipole sources," Journal of Sound and Vibration, Vol. 330, 2011, pp. 5835-5851.

${ }^{9}$ Dougherty, R. P., "Improved Generalized Inverse Beamforming for Jet Noise," 17th AIAA/CEAS Aeroacoustics Conference, AIAA-2011-2769, Portland, OR, June 2011.

${ }^{10}$ Bendat, J. S. and Piersol, A. G., Random Data Analysis and Measurement Procedures, chap. 5, John Wiley \& Sons, Inc., New York, NY, 3rd ed., 2000, p. 146.

${ }^{11}$ Johnson, D. H. and Dudgeon, D. E., Array Signal Processing: Concepts and Techniques, chap. 3, Prentice Hall, Englewood Cliffs, NJ, 1993, p. 92.

${ }^{12}$ Capon, J., "Signal processing and frequency-wavenumber spectrum analysis for a large aperture seismic array," Methods in Computational Physics, Vol. 13, 1973, pp. 1-59.

${ }^{13}$ Dougherty, R. P., Aeroacoustic Measurements, chap. Beamforming in Acoustic Testing, Springer-Verlag, Berlin, Heidelberg \& New York, 2002.

${ }^{14}$ Atkinson, K. E., An Introduction to Numerical Analysis, John Wiley \& Sons, Inc., 2nd ed., 1989.

${ }^{15}$ Frigo, M. and Johnson, S. G., "The Design and Implementation of FFTW3," Proceedings of the IEEE, Vol. 93, No. 2, 2005, pp. 216-231.

${ }^{16}$ Bahr, C., Zawodny, N. S., Bertolucci, B., Woolwine, K., Liu, F., Li, J., Sheplak, M., and Cattafesta, L., "Measurement of Phased Array Point Spread Functions for use with Beamforming," 17th AIAA/CEAS Aeroacoustics Conference, AIAA-20112767, Portland, OR, June 2011.

${ }^{17}$ CUDA Toolkit 4.1, CUFFT Library Programming Guide, NVIDIA Corporation, January 2012.
} 
${ }^{18}$ Franchetti, F. and Püschel, M., "High-Performance Pruned FFT Implementations," Proc. International Conference on Acoustics, Speech, and Signal Processing, 2009, pp. 549-552.

${ }^{19}$ Barrows, B. E., Teixeira, F. L., and Kong, J. A., "Fast Algorithm for Matrix-Vector Multiply of Asymmetric Multilevel Block-Toeplitz Matrices in 3-D Scattering," Microwave and Optical Technology Letters, Vol. 31, No. 1, October 2001 , pp. $28-32$.

${ }^{20}$ Brandt, A., "Multi-Level Adaptive Solutions to Boundary-Value Problems," Mathematics of Computation, Vol. 31, No. 138, April 1977, pp. 333-390.

${ }^{21}$ Yuan, L., Sun, J., Quan, L., and Shum, H.-Y., "Progressive Inter-scale and Intra-scale Non-blind Image Deconvolution," ACM Transactions on Graphics, Vol. 27, No. 3, Article 74, 2008.

${ }^{22}$ Español, M. I., Multilevel Methods for Discrete Ill-Posed Problems: Application to Deblurring, Ph.D. thesis, Tufts University, May 2009.

${ }^{23}$ Millar, J. and Bancroft, J. C., "Multigrid Deconvolution of Seismic Data," 2004 SEG Annual Meeting, October 10 - 15, 2004.

${ }^{24}$ Underbrink, J. R., Practical Considerations in Focused Array Design for Passive Broad-Band Source Mapping Applications, Master's thesis, The Pennsylvania State University, State College, PA, 1995.

${ }^{25}$ Johnson, D. H. and Dudgeon, D. E., Array Signal Processing: Concepts and Techniques, chap. 4, Prentice Hall, Englewood Cliffs, NJ, 1993, p. 176.

${ }^{26}$ Bahr, C., Li, J., and Cattafesta, L., "Aeroacoustic Measurements in Open-jet Wind Tunnels - An Evaluation of Methods Applied to Trailing Edge Noise," 17th AIAA/CEAS Aeroacoustics Conference, AIAA-2011-2771, Portland, OR, June 2011. 